\title{
STOCHASTIC TWO-SCALE CONVERGENCE OF AN INTEGRAL FUNCTIONAL
}

\author{
MAMADOU SANGO AND JEAN LOUIS WOUKENG
}

\begin{abstract}
In this paper we discuss the concept of stochastic two-scale convergence, which is appropriate to solve coupled -periodic and stochastic- homogenization problems. This concept is a combination of both well-known two-scale convergence and stochastic two-scale convergence in the mean schemes, and is a generalization of the said previous methods. By way of illustration we apply it to solve a homogenization problem related to an integral functional with convex integrand. This problematic relies on the notion of dynamical system which is our basic tool.
\end{abstract}

\section{INTRODUCTION}

The two-scale convergence method by Nguetseng [23] has proved very efficient to handle periodic homogenization problems. It then generated a great number of research activities that increases over time as shown by the vast existing literature to date; see e.g., $[2,3,5,6,20,37,38,39]$ and the references therein. However, being strictly limited to periodic structures, it quickly showed its inadequacy as far as the non periodic phenomena are concerned, since in nature, few physical phenomena have in fact a periodic behaviour. Furthermore these phenomena are often subjected to randomness. To overcome this inadequacy, Bourgeat et al. [10] introduced the stochastic two-scale convergence in the mean's method. It has helped to go from deterministic periodic homogenization theory to stochastic homogenization theory, much closer to the reality of natural and physical phenomena. It is worth pointing out that in between the deterministic periodic and stochastic homogenization theories, there exists a recent general deterministic homogenization theory which was built up recently in [24], and which has just been improved in [26]. We also draw the attention of the reader to the papers [41] and [7]. In the first paper [41] a systematic treatment of the stochastic two-scale convergence with respect to invariant probability measures is done. It is to be noted that in the said-paper the authors consider probability spaces defined on compact metric spaces. In [7] the authors propose an alternative approach to stochastic homogenization by using diffeomorphisms to perturb the microscale.

In this work we rely on the previous two convergence methods (see [23] and [10]) to propose a general method of solving coupled - periodic and stochastic - homogenization problems. Our method, the stochastic two-scale convergence method combining both two-scale convergence and stochastic two-scale convergence in the mean schemes is a straightforward generalization of the previous method and seems to be appropriate for natural phenomena since most of these phenomena behave

2000 Mathematics Subject Classification. 35B27, 35B40, 37A05, 37A55.

Key words and phrases. Dynamical system, homogenization, stochastic two-scale convergence. 
randomly in some scales, and deterministically in other scales. Our multiscale approach is motivated by the fact that usual monoscale approach has proven to be inadequate because of prohibitively large number of variables involved in each physical problem. One can also give at least two reasons quite natural. Firstly, a scale can not be at the same time deterministic and random. Secondly, the application of our results to natural phenomena. This second reason is the most important; in fact let us be more precise and give an extra motivation for the choice in this case, which motivation arises from the following quite simple example. It is known that the human body is an example of medium that presents both a random and deterministic behaviour. In particular it contains an exciting class of nonlinear materials presenting microstructures like myocardium, arterials walls, cartilage, muscles etc. These biological materials are characterized by hierarchical ordering of microstructures, ranging randomly from nanoscale to macroscale. The understanding of these materials requires a proper study, which can be provided by means of deterministic-stochastic homogenization theory. That is why it becomes urgent to develop systematic method and approach for multiscale problems. This is the aim of the present work.

Returning to our original issue, we aim at providing a framework suitable to handle both deterministic and stochastic homogenization problems. Our approach is based on the well-known theory of dynamical systems.

The organization of the paper is as follows. In Section 2, we give some preliminaries about the dynamical systems. In Section 3, we state the concept of stochastic two-scale convergence and we prove some compactness results. In Section 4, we apply the results of Section 3 to solve a mixed periodic-stochastic homogenization problem related to an integral functional with convex integrand.

Unless otherwise specified, vector spaces throughout are assumed to be real vector spaces, and scalar functions are assumed to take real values. We shall always assume that the numerical spaces $\mathbb{R}^{m}$ and their open sets are each equipped with the Lebesgue measure.

\section{PRELiminaries ON DYNAMiCAL SYSTEMS}

We begin by recalling the definition of the notion of a dynamical system. Let $(\Omega, \mathcal{M}, \mu)$ denote a probability space. An $N$-dimensional dynamical system on $\Omega$ is a family of invertible mappings $T(x): \Omega \rightarrow \Omega, x \in \mathbb{R}^{N}$, such that the following conditions hold:

(i) (Group property) $T(0)=i d_{\Omega}$ and $T(x+y)=T(x) \circ T(y)$ for all $x, y \in \mathbb{R}^{N}$;

(ii) (Invariance) The mappings $T(x): \Omega \rightarrow \Omega$ are measurable and $\mu$-measure preserving, i.e., $\mu(T(x) F)=\mu(F)$ for each $x \in \mathbb{R}^{N}$ and every $F \in \mathcal{M}$;

(iii) (Measurability) For each $F \in \mathcal{M}$, the set $\left\{(x, \omega) \in \mathbb{R}^{N} \times \Omega: T(x) \omega \in F\right\}$ is measurable with respect to the product $\sigma$-algebra $\mathcal{L} \otimes \mathcal{M}$, where $\mathcal{L}$ is the $\sigma$-algebra of Lebesgue measurable sets.

If $\Omega$ is a compact topological space, by a continuous $N$-dimensional dynamical system on $\Omega$ we mean any family of mappings $T(x): \Omega \rightarrow \Omega, x \in \mathbb{R}^{N}$, satisfying the above group property (i) and the following condition: The mapping $(x, \omega) \mapsto T(x) \omega$ is continuous from $\mathbb{R}^{N} \times \Omega$ to $\Omega$. 
Let $1 \leq p \leq \infty$. An $N$-dimensional dynamical system $T(x): \Omega \rightarrow \Omega$ induces a $N$-parameter group of isometries $U(x): L^{p}(\Omega) \rightarrow L^{p}(\Omega)$ defined by

$$
(U(x) f)(\omega)=f(T(x) \omega), f \in L^{p}(\Omega)
$$

which is strongly continuous, i.e., $U(x) f \rightarrow f$ in $L^{p}(\Omega)$ as $x \rightarrow 0$; see [18, p. 223] or $\left[28\right.$, p. 131]. We denote by $D_{i, p}(1 \leq i \leq N)$ the generator of $U(x)$ along the $i$ th coordinate direction, and by $\mathcal{D}_{i, p}$ its domain. Thus, for $f \in L^{p}(\Omega), f$ is in $\mathcal{D}_{i, p}$ if and only if the limit $D_{i, p} f$ defined by

$$
D_{i, p} f(\omega)=\lim _{\tau \rightarrow 0} \frac{f\left(T\left(\tau e_{i}\right) \omega\right)-f(\omega)}{\tau}
$$

exists strongly in $L^{p}(\Omega)$, where $e_{i}$ denotes the vector $\left(\delta_{i j}\right)_{1 \leq j \leq N}, \delta_{i j}$ being the Kronecker symbol. One can naturally define higher order derivatives by setting $D_{p}^{\alpha}=D_{1, p}^{\alpha_{1}} \cdots D_{N, p}^{\alpha_{N}}$ for $\alpha=\left(\alpha_{1}, \ldots, \alpha_{N}\right) \in \mathbb{N}^{N}$.

Now we need to define the stochastic analog of the smooth functions on $\mathbb{R}^{N}$. To this end, we set $\mathcal{D}_{p}(\Omega)=\cap_{i=1}^{N} \mathcal{D}_{i, p}$ and define

$$
\mathcal{D}_{p}^{\infty}(\Omega)=\left\{f \in L^{p}(\Omega): D_{p}^{\alpha} f \in \mathcal{D}_{p}(\Omega) \text { for all } \alpha \in \mathbb{N}^{N}\right\} .
$$

It is a fact that each element of $\mathcal{D}_{\infty}^{\infty}(\Omega)$ possesses stochastic derivatives of any order that are bounded. So as in [4] we denote it by the suggestive symbol $\mathcal{C}^{\infty}(\Omega)$, and it can be shown that $\mathcal{C}^{\infty}(\Omega)$ is dense in $L^{p}(\Omega), 1 \leq p<\infty$. At this level, one can naturally define the concept of stochastic distribution: by a stochastic distribution on $\Omega$ is meant any continuous linear mapping from $\mathcal{C}^{\infty}(\Omega)$ to the real field $\mathbb{R}$. We recall that $\mathcal{C}^{\infty}(\Omega)$ is endowed with its natural topology defined by the family of seminorms $N_{n}(f)=\sup _{|\alpha| \leq n} \sup _{\omega \in \Omega}\left|D_{\infty}^{\alpha} f(\omega)\right|$ (where $|\alpha|=\alpha_{1}+\ldots+\alpha_{N}$ for $\left.\alpha=\left(\alpha_{1}, \ldots, \alpha_{N}\right) \in \mathbb{N}^{N}\right)$. We denote the space of stochastic distributions by $\left(\mathcal{C}^{\infty}(\Omega)\right)^{\prime}$. One can also define the stochastic weak derivative of $f \in\left(\mathcal{C}^{\infty}(\Omega)\right)^{\prime}$ as follows: For any $\alpha \in \mathbb{N}^{N}, D^{\alpha} f$ stands for the stochastic distribution defined by

$$
\left(D^{\alpha} f\right)(\phi)=(-1)^{|\alpha|} f\left(D^{\alpha} \phi\right) \quad \forall \phi \in \mathcal{C}^{\infty}(\Omega) .
$$

As $\mathcal{C}^{\infty}(\Omega)$ is dense in $L^{p}(\Omega)(1 \leq p<\infty)$, it is immediate that $L^{p}(\Omega) \subset\left(\mathcal{C}^{\infty}(\Omega)\right)^{\prime}$ so that one may define the stochastic weak derivative of any $f \in L^{p}(\Omega)$, and it verifies the following functional equation: $\left(D^{\alpha} f\right)(\phi)=(-1)^{|\alpha|} \int_{\Omega} f D^{\alpha} \phi d \mu$ for all $\phi \in \mathcal{C}^{\infty}(\Omega)$. In particular, for $f \in \mathcal{D}_{i, p}$ we have $-\int_{\Omega} f D_{i, \infty} \phi d \mu=\int_{\Omega} \phi D_{i, p} f d \mu$ for all $\phi \in \mathcal{C}^{\infty}(\Omega)$ so that we may identify $D_{i, p} f$ with $D^{\alpha_{i}} f$, where $\alpha_{i}=\left(\delta_{i j}\right)_{1<j<N}$. Conversely, if $f \in L^{p}(\Omega)$ is such that there exists $f_{i} \in L^{p}(\Omega)$ with $\left(D^{\alpha_{i}} f\right)(\phi)=$ $-\int_{\Omega} f_{i} \phi d \mu$ for all $\phi \in \mathcal{C}^{\infty}(\Omega)$, then $f \in \mathcal{D}_{i, p}$ and $D_{i, p} f=f_{i}$. Therefore, endowing $\mathcal{D}_{p}(\Omega)$ with the natural graph norm

$$
\|f\|_{\mathcal{D}_{p}(\Omega)}^{p}=\|f\|_{L^{p}(\Omega)}^{p}+\sum_{i=1}^{N}\left\|D_{i, p} f\right\|_{L^{p}(\Omega)}^{p}\left(f \in \mathcal{D}_{p}(\Omega)\right)
$$

we obtain a Banach space representing the stochastic generalization of the Sobolev spaces $W^{1, p}\left(\mathbb{R}^{N}\right)$, and so, we denote it by $W^{1, p}(\Omega)$.

Now, returning to the general setting of dynamical systems, we recall that a function $f \in L^{p}(\Omega)$ is said to be invariant for $T$ (relative to $\mu$ ) if for any $x \in \mathbb{R}^{N}$, $f \circ T(x)=f \mu$-a.e. on $\Omega$. We denote by $I_{n v}^{p}(\Omega)$ the set of functions in $L^{p}(\Omega)$ that are invariant for $T$. The set $I_{n v}^{p}(\Omega)$ is a closed vector subspace of $L^{p}(\Omega)$. The dynamical system $T$ is said to be ergodic if every invariant function is $\mu$-equivalent to a constant. We have the following very useful properties for functions in $L^{1}(\Omega)$. 
(P1) For $f \in \mathcal{D}_{1}^{\infty}(\Omega)$, and for $\mu$-a.e. $\omega \in \Omega$, the function $x \mapsto f(T(x) \omega)$ is in $\mathcal{C}^{\infty}\left(\mathbb{R}^{N}\right)$ and further $D_{x}^{\alpha} f(T(x) \omega)=\left(D_{1}^{\alpha} f\right)(T(x) \omega)$ for any $\alpha \in \mathbb{N}^{N}$.

(P2) For $f \in L^{1}(\Omega)$, we have $f \in I_{n v}^{1}(\Omega)$ if and only if $D_{i, 1} f=0$ for each $1 \leq i \leq N$.

Let $1<p<\infty$. Thanks to (P2) above, one can easily check that, for $f \in L^{p}(\Omega)$, $f$ is in $I_{n v}^{p}(\Omega)$ if and only if $D_{i, p} f=0$ for all $1 \leq i \leq N$. So if we endow $\mathcal{C}^{\infty}(\Omega)$ with the seminorm

$$
\|u\|_{\#, p}^{p}=\sum_{i=1}^{N}\left\|D_{i, p} u\right\|_{L^{p}(\Omega)}^{p} \quad\left(u \in \mathcal{C}^{\infty}(\Omega)\right)
$$

we obtain a locally convex space which is generally not complete. We denote by $\mathcal{W}^{1, p}(\Omega)$ the completion of $\mathcal{C}^{\infty}(\Omega)$ with respect to the seminorm $\|\cdot\|_{\#, p}$, and we denote by $I_{p}$ the canonical mapping of $\mathcal{C}^{\infty}(\Omega)$ into its completion $\mathcal{W}^{1, p}(\Omega)$. The following property is obtained through the theory of completion of uniform spaces; see, e.g., [8, Chap. II, Sect. 3, no 7].

The gradient operator $D_{\omega, p}=\left(D_{1, p}, \ldots, D_{N, p}\right): \mathcal{C}^{\infty}(\Omega) \rightarrow L^{p}(\Omega)^{N}$ extends by continuity to a unique mapping $\bar{D}_{\omega, p}=\left(\bar{D}_{1, p}, \ldots, \bar{D}_{N, p}\right): \mathcal{W}^{1, p}(\Omega) \rightarrow L^{p}(\Omega)^{N}$ with the properties

$$
D_{i, p}=\bar{D}_{i, p} \circ I_{p}
$$

and

$$
\|u\|_{\mathcal{W}^{1, p}(\Omega)} \equiv\|u\|_{\#, p}=\left(\sum_{i=1}^{N}\left\|\bar{D}_{i, p} u\right\|_{L^{p}(\Omega)}^{p}\right)^{1 / p} \text { for } u \in \mathcal{W}^{1, p}(\Omega) .
$$

Moreover, the mapping $\bar{D}_{\omega, p}$ is an isometric embedding of $\mathcal{W}^{1, p}(\Omega)$ into a closed subspace of $L^{p}(\Omega)^{N}$, so that the Banach space $\mathcal{W}^{1, p}(\Omega)$ is reflexive. By duality we define the operator $\operatorname{div}_{\omega, p^{\prime}}: L^{p^{\prime}}(\Omega)^{N} \rightarrow\left(\mathcal{W}^{1, p}(\Omega)\right)^{\prime}\left(p^{\prime}=p /(p-1)\right)$ by

$$
\left\langle\operatorname{div}_{\omega, p^{\prime}} u, w\right\rangle=-\left\langle u, \bar{D}_{\omega, p} w\right\rangle \text { for all } w \in \mathcal{W}^{1, p}(\Omega) \text {. }
$$

The operator $\operatorname{div}_{\omega, p^{\prime}}$ just defined extends the natural divergence operator defined in $\mathcal{C}^{\infty}(\Omega)$ since for all $f \in \mathcal{C}^{\infty}(\Omega)$ we have $D_{i, p} f=\bar{D}_{i, p}\left(I_{p}(f)\right)$.

The following result will be of great interest in the next sections.

Proposition 1. Let $v \in L^{p}(\Omega)^{N}$ satisfy

$$
\int_{\Omega} v \cdot g d \mu=0 \text { for all } g \in \mathcal{V}_{\mathrm{div}}=\left\{f \in \mathcal{C}^{\infty}(\Omega)^{N}: \operatorname{div}_{\omega, p^{\prime}} f=0\right\} .
$$

Then there exists $u \in \mathcal{W}^{1, p}(\Omega)$ such that $v=\bar{D}_{\omega, p} u$.

Proof. We need to check the following: (1) $\operatorname{div}_{\omega, p^{\prime}}$ is closed; $(2)\left(\operatorname{div}_{\omega, p^{\prime}}\right)^{*}=-\bar{D}_{\omega, p}$ where $\left(\operatorname{div}_{\omega, p^{\prime}}\right)^{*}$ is the adjoint operator of $\operatorname{div}_{\omega, p^{\prime}} ;(3) \operatorname{Range}\left(\bar{D}_{\omega, p}\right)$ is closed in $L^{p}(\Omega)^{N}$ and finally, (4) $v$ is orthogonal to the kernel of $\operatorname{div}_{\omega, p^{\prime}}$. Indeed (1)-(3) will yield Range $\left(\bar{D}_{\omega, p}\right)=\left(\operatorname{ker}\left(\operatorname{div}_{\omega, p^{\prime}}\right)\right)^{\perp}$ by a well-known result (see, e.g., [30, Chap. 13]) where $\left(\operatorname{ker}\left(\operatorname{div}_{\omega, p^{\prime}}\right)\right)^{\perp}$ denote the orthogonal complement of $\operatorname{ker}\left(\operatorname{div}_{\omega, p^{\prime}}\right)$, and finally the proposition will follow at once from (4). So let us check them.

(1) is trivial, (2) is a mere consequence of the definition of $\operatorname{div}_{\omega, p^{\prime}}$. As for (3), if $v_{n}=\bar{D}_{\omega, p} u_{n} \in \operatorname{Range}\left(\bar{D}_{\omega, p}\right)$ is such that $v_{n} \rightarrow v$ in $L^{p}(\Omega)^{N}$, then $\left(u_{n}\right)_{n}$ is a Cauchy sequence in $\mathcal{W}^{1, p}(\Omega)$ and so, converges in $\mathcal{W}^{1, p}(\Omega)$ towards some $u \in \mathcal{W}^{1, p}(\Omega)$, that is, $\bar{D}_{\omega, p} u_{n} \rightarrow \bar{D}_{\omega, p} u$ in $L^{p}(\Omega)^{N}$, hence $v=\bar{D}_{\omega, p} u$. Finally for (4) it suffices to 
show that $\mathcal{V}_{\text {div }}$ is dense in $\operatorname{ker}\left(\operatorname{div}_{\omega, p^{\prime}}\right)$. To see this, let $g \in \operatorname{ker}\left(\operatorname{div}_{\omega, p^{\prime}}\right)$; arguing as in the proof of [10, Lemma 2.3 (b)] there exists a sequence $\left(g_{n}\right)_{n} \subset \mathcal{V}_{\text {div }}$ such that $g_{n} \rightarrow g$ in $L^{p}(\Omega)^{N}$. The proof is complete.

We end this section with some definitions. Let $f$ be a measurable function in $\Omega$; for a fixed $\omega \in \Omega$ the function $x \mapsto f(T(x) \omega), x \in \mathbb{R}^{N}$, is called a realization of $f$ and the mapping $(x, \omega) \mapsto f(T(x) \omega)$ is called a stationary process. The process is said to be stationary ergodic if the dynamical system $T$ is ergodic.

In the forthcoming sections we will adopt the following notation: $\bar{D}_{\omega}$ will stand for $\bar{D}_{\omega, p}$, and, $\bar{D}_{i, p}$ (resp. $D_{i, p}$ ) will be denoted by $\bar{D}_{i, \omega}$ (resp. $\left.D_{i, \omega}\right)$ if there is no danger of confusion.

\section{The STOCHASTIC TWO-SCALE CONVERGENCE}

In this section we define the concept of stochastic two-scale convergence which is the generalization of both two-scale convergence in the mean (of Bourgeat et al. [10]) and two scale convergence (of Nguetseng [23]). In all that follows, $Q$ is an open subset of $\mathbb{R}^{N}$ and $\left\{T(y): y \in \mathbb{R}^{N}\right\}$ denotes an $N$-dimensional dynamical system acting on the probability space $(\Omega, \mathcal{M}, \mu)$. Points in $\Omega$ are denoted by $\omega$. Next, let $\varepsilon_{1}$ and $\varepsilon_{2}$ be two functions of $\varepsilon>0$ satisfying the condition $0<\varepsilon_{1}, \varepsilon_{2} \rightarrow 0$ as $\varepsilon \rightarrow 0$ together with the well-separatedness condition $\varepsilon_{2} / \varepsilon_{1} \rightarrow 0$ as $\varepsilon \rightarrow 0$. We end this with some notations. Let $Y=(0,1)^{N}$ and let $F\left(\mathbb{R}^{N}\right)$ be a given function space. We denote by $F_{\text {per }}(Y)$ the space of functions in $F_{\text {loc }}\left(\mathbb{R}^{N}\right)$ that are $Y$-periodic, and by $F_{\#}(Y)$ those functions in $F_{\text {per }}(Y)$ with mean value zero. As special cases, $\mathcal{D}_{\text {per }}(Y)$ denotes the space $\mathcal{C}_{\mathrm{per}}^{\infty}(Y)$ while $\mathcal{D}_{\#}(Y)$ stands for the space of those functions in $\mathcal{D}_{\text {per }}(Y)$ with mean value zero. $\mathcal{D}_{\text {per }}^{\prime}(Y)$ stands for the topological dual of $\mathcal{D}_{\text {per }}(Y)$ which can be identified with the space of periodic distributions in $\mathcal{D}^{\prime}\left(\mathbb{R}^{N}\right)$.

Definition 1. Let $\left(u_{\varepsilon}\right)_{\varepsilon>0}$ be a bounded sequence in $L^{p}(Q \times \Omega)(1 \leq p<\infty)$. The sequence $\left(u_{\varepsilon}\right)_{\varepsilon>0}$ is said to weakly stochastically two-scale converge in $L^{p}(Q \times \Omega)$ to some $u_{0} \in L^{p}\left(Q \times \Omega ; L_{\mathrm{per}}^{p}(Y)\right)$ if as $\varepsilon \rightarrow 0$, we have

$$
\int_{Q \times \Omega} u_{\varepsilon}(x, \omega) f\left(x, T\left(\frac{x}{\varepsilon_{1}}\right) \omega, \frac{x}{\varepsilon_{2}}\right) d x d \mu \rightarrow \iint_{Q \times \Omega \times Y} u_{0}(x, \omega, y) f(x, \omega, y) d x d \mu d y
$$

for every $f \in \mathcal{C}_{0}^{\infty}(Q) \otimes \mathcal{C}^{\infty}(\Omega) \otimes \mathcal{C}_{\text {per }}^{\infty}(Y)$. We express this by writing $u_{\varepsilon} \rightarrow u_{0}$ stoch. in $L^{p}(Q \times \Omega)$-weak $2 s$.

We recall that $\mathcal{C}_{0}^{\infty}(Q) \otimes \mathcal{C}^{\infty}(\Omega) \otimes \mathcal{C}_{\text {per }}^{\infty}(Y)$ is the space of functions of the form

$$
f(x, \omega, y)=\sum_{\text {finite }} \varphi_{i}(x) \psi_{i}(\omega) g_{i}(y), \quad(x, \omega, y) \in Q \times \Omega \times \mathbb{R}^{N}
$$

with $\varphi_{i} \in \mathcal{C}_{0}^{\infty}(Q), \psi_{i} \in \mathcal{C}^{\infty}(\Omega)$ and $g_{i} \in \mathcal{C}_{\text {per }}^{\infty}(Y)$. Such functions are dense in $\mathcal{C}_{0}^{\infty}(Q) \otimes L^{p^{\prime}}(\Omega) \otimes \mathcal{C}_{\text {per }}^{\infty}(Y)\left(p^{\prime}=p /(p-1)\right.$ for $1<p<\infty$, since $\mathcal{C}^{\infty}(\Omega)$ is dense in $\left.L^{p^{\prime}}(\Omega)\right)$ and hence in $\mathcal{K}\left(Q ; L^{p^{\prime}}(\Omega)\right) \otimes \mathcal{C}_{\text {per }}^{\infty}(Y)\left(\mathcal{K}\left(Q ; L^{p^{\prime}}(\Omega)\right)\right.$ being the space of continuous functions of $Q$ into $L^{p^{\prime}}(\Omega)$ with compact support containing in $Q$; see e.g., [9, Chap. III, Sect. 1, Proposition 5] for the denseness result). As $\mathcal{K}\left(Q ; L^{p^{\prime}}(\Omega)\right)$ is dense in $L^{p^{\prime}}\left(Q ; L^{p^{\prime}}(\Omega)\right)=L^{p^{\prime}}(Q \times \Omega)$ and $L^{p^{\prime}}(Q \times \Omega) \otimes \mathcal{C}_{\text {per }}^{\infty}(Y)$ is dense in $L^{p^{\prime}}\left(Q \times \Omega ; \mathcal{C}_{\text {per }}(Y)\right)$, the uniqueness of the stochastic two-scale limit is ensured. 
Remark 1. For a better understanding of our results and a simple presentation of the paper, we have restricted ourselves to just one deterministic scale and one random scale in Definition 1. The more general case of several scales of each type can be considered, and the results in that case follow by repeating the arguments that will be used to prove the ones in the present setting, as one can easily see it in the sequel.

Before continuing our study, we need to make a comparison between the stochastic two-scale convergence and other existing convergence methods closed to it. For that, we must first state these convergence schemes:

(1) A sequence $\left(u_{\varepsilon}\right)_{\varepsilon>0} \subset L^{p}(Q)(1 \leq p<\infty)$ is said to weakly two-scale converge in $L^{p}(Q)$ to some $v_{0} \in L^{p}\left(Q ; L_{\mathrm{per}}^{p}(Y)\right)$ if as $\varepsilon \rightarrow 0$, we have

$$
\int_{Q} u_{\varepsilon}(x) f\left(x, \frac{x}{\varepsilon_{2}}\right) d x \rightarrow \iint_{Q \times Y} v_{0}(x, y) f(x, y) d x d y
$$

for every $f \in L^{p^{\prime}}\left(Q ; \mathcal{C}_{\text {per }}(Y)\right)\left(1 / p^{\prime}=1-1 / p\right)$.

(2) A sequence $\left(u_{\varepsilon}\right)_{\varepsilon>0} \subset L^{p}(Q \times \Omega)(1 \leq p<\infty)$ is said to stochastically two-scale converge in the mean to some $v_{0} \in L^{p}(Q \times \Omega)$ if as $\varepsilon \rightarrow 0$, we have

$$
\iint_{Q \times \Omega} u_{\varepsilon}(x, \omega) f\left(x, T\left(\frac{x}{\varepsilon_{1}}\right) \omega\right) d x d \mu \rightarrow \iint_{Q \times \Omega} v_{0}(x, \omega) f(x, \omega) d x d \mu
$$

for all admissible functions (in the sense of [10, Section 3]) $f \in L^{p^{\prime}}(Q \times \Omega)$. We denote it by $u_{\varepsilon} \rightarrow u_{0}$ stoch. in $L^{p}(Q \times \Omega)$-weak.

It is very important to note that both the above definitions (3.2) and (3.3) imply the boundedness of the sequence $u_{\varepsilon}$ either in $L^{p}(Q)$ or in $L^{p}(Q \times \Omega)$, accordingly. With this in mind, we see that the stochastic two-scale convergence method generalizes the above two convergence methods. Indeed, if in (3.1) we take $f \in \mathcal{C}_{0}^{\infty}(Q) \otimes \mathcal{C}^{\infty}(\Omega)$, that is $f$ is constant with respect to $y \in \mathbb{R}^{N}$, and next using the density of the latter space in $L^{p^{\prime}}(Q \times \Omega)$, then (3.1) reads as (3.3) with $v_{0}(x, \omega)=\int_{Y} u_{0}(x, \omega, y) d y$ by choosing in $L^{p^{\prime}}(Q \times \Omega)$ admissible functions. If besides we take in $(3.1) f \in \mathcal{C}_{0}^{\infty}(Q) \otimes \mathcal{C}_{\text {per }}^{\infty}(Y)$, that is $f$ not depending upon the random variable $\omega$ and further if we choose $u_{\varepsilon}$ not depending on $\omega$, then using the density of $\mathcal{C}_{0}^{\infty}(Q) \otimes \mathcal{C}_{\text {per }}^{\infty}(Y)$ in $L^{p^{\prime}}\left(Q ; \mathcal{C}_{\text {per }}(Y)\right)$ we readily get $(3.2)$ with $v_{0}(x, y)=\int_{\Omega} u_{0}(x, \omega, y) d \mu$.

The following result is easily verified; its proof is left to the reader.

Proposition 2. Let $\left(u_{\varepsilon}\right)_{\varepsilon>0}$ be a sequence in $L^{p}(Q \times \Omega)$. If $u_{\varepsilon} \rightarrow u_{0}$ stoch. in $L^{p}(Q \times \Omega)$-weak $2 s$, then $\left(u_{\varepsilon}\right)_{\varepsilon>0}$ stochastically two-scale converges in the mean towards $v_{0}(x, \omega)=\int_{Y} u_{0}(x, \omega, y) d y$ and

$$
\int_{\Omega} u_{\varepsilon}(\cdot, \omega) \psi(\omega) d \mu \rightarrow \iint_{\Omega \times Y} u_{0}(\cdot, \omega, y) \psi(\omega) d \mu d y \text { in } L^{1}(Q) \text {-weak } \forall \psi \in I_{n v}^{p^{\prime}}(\Omega) \text {. }
$$

In order to state and prove the first compactness result of this section, we need one preliminary result. In what follows, $Q$ is assumed to be bounded.

Proposition 3. Let $f \in \mathcal{K}\left(Q ; \mathcal{C}_{\text {per }}\left(Y ; \mathcal{C}^{\infty}(\Omega)\right)\right)$. Then, as $\varepsilon \rightarrow 0$,

$$
\int_{Q \times \Omega} f\left(x, T\left(\frac{x}{\varepsilon_{1}}\right) \omega, \frac{x}{\varepsilon_{2}}\right) d x d \mu \rightarrow \iint_{Q \times \Omega \times Y} f(x, \omega, y) d x d \mu d y .
$$


Proof. Since $\mathcal{C}_{0}^{\infty}(Q) \otimes \mathcal{C}^{\infty}(\Omega) \otimes \mathcal{C}_{\text {per }}(Y)$ is dense in $\mathcal{K}\left(Q ; \mathcal{C}_{\text {per }}\left(Y ; \mathcal{C}^{\infty}(\Omega)\right)\right)$, we first check (3.4) for $f$ in $\mathcal{C}_{0}^{\infty}(Q) \otimes \mathcal{C}^{\infty}(\Omega) \otimes \mathcal{C}_{\text {per }}(Y)$. For that, it is sufficient to do it for $f$ under the form $f(x, \omega, y)=\varphi(x) \psi(\omega) g(y)$ with $\varphi \in \mathcal{C}_{0}^{\infty}(Q), \psi \in \mathcal{C}^{\infty}(\Omega)$ and $g \in \mathcal{C}_{\text {per }}(Y)$. But for such a $f$ we have

$$
\begin{aligned}
\int_{Q \times \Omega} f\left(x, T\left(\frac{x}{\varepsilon_{1}}\right) \omega, \frac{x}{\varepsilon_{2}}\right) d x d \mu & =\int_{Q}\left(\int_{\Omega} \psi\left(T\left(\frac{x}{\varepsilon_{1}}\right) \omega\right) d \mu\right) \varphi(x) g\left(\frac{x}{\varepsilon_{2}}\right) d x \\
& =\int_{Q}\left(\int_{\Omega} \psi(\omega) d \mu\right) \varphi(x) g\left(\frac{x}{\varepsilon_{2}}\right) d x \\
& =\left(\int_{\Omega} \psi(\omega) d \mu\right) \int_{Q} \varphi(x) g\left(\frac{x}{\varepsilon_{2}}\right) d x
\end{aligned}
$$

where the second equality above is due to the Fubini's theorem and to the fact that the measure $\mu$ is invariant under the maps $T(y)$. But, as $\varepsilon \rightarrow 0$, we have the following well-known convergence result:

$$
\int_{Q} \varphi(x) g\left(\frac{x}{\varepsilon_{2}}\right) d x \rightarrow \iint_{Q \times Y} \varphi(x) g(y) d x d y \text { as } \varepsilon \rightarrow 0 .
$$

Hence the sequence $\int_{Q \times \Omega} f\left(x, T\left(\frac{x}{\varepsilon_{1}}\right) \omega, \frac{x}{\varepsilon_{2}}\right) d x d \mu$ converges towards

$$
\left(\int_{\Omega} \psi(\omega) d \mu\right)\left(\iint_{Q \times Y} \varphi(x) g(y) d x d y\right)=\iint_{Q \times \Omega \times Y} f(x, \omega, y) d x d \mu d y .
$$

Next, let $f \in \mathcal{K}\left(Q ; \mathcal{C}_{\text {per }}\left(Y ; \mathcal{C}^{\infty}(\Omega)\right)\right)$ and let $\eta>0$ be arbitrarily fixed. By a density argument we choose $\phi$ in $\mathcal{C}_{0}^{\infty}(Q) \otimes \mathcal{C}^{\infty}(\Omega) \otimes \mathcal{C}_{\text {per }}(Y)$ such that $\|f-\phi\|_{\infty} \leq$ $\eta /(3|Q|),|Q|$ denoting the Lebesgue volume of $Q$. By the decomposition

$$
\begin{aligned}
\int_{Q \times \Omega} f^{\varepsilon} d x d \mu-\iint_{Q \times \Omega \times Y} f d x d \mu d y= & \int_{Q \times \Omega}\left(f^{\varepsilon}-\phi^{\varepsilon}\right) d x d \mu \\
& +\int_{Q \times \Omega} \phi^{\varepsilon} d x d \mu-\iint_{Q \times \Omega \times Y} \phi d x d \mu d y \\
& +\iint_{Q \times \Omega \times Y}(\phi-f) d x d \mu d y
\end{aligned}
$$

it follows readily that there exists $\varepsilon_{0}>0$ such that

$$
\left|\int_{Q \times \Omega} f^{\varepsilon} d x d \mu-\iint_{Q \times \Omega \times Y} f d x d \mu d y\right| \leq \eta \text { for } 0<\varepsilon \leq \varepsilon_{0} .
$$

This completes the proof.

Now, since $\mathcal{K}\left(Q ; \mathcal{C}_{\text {per }}\left(Y ; \mathcal{C}^{\infty}(\Omega)\right)\right)$ is a Banach algebra, it is easily shown that, for $1 \leq p<\infty$ we have $|f|^{p} \in \mathcal{K}\left(Q ; \mathcal{C}_{\text {per }}\left(Y ; \mathcal{C}^{\infty}(\Omega)\right)\right)$ whenever $f \in \mathcal{K}\left(Q ; \mathcal{C}_{\text {per }}\left(Y ; \mathcal{C}^{\infty}(\Omega)\right)\right)$, so that, as $\varepsilon \rightarrow 0$,

$$
\int_{Q \times \Omega}\left|f^{\varepsilon}\right|^{p} d x d \mu \rightarrow \iint_{Q \times \Omega \times Y}|f|^{p} d x d \mu d y .
$$

The above convergence result will be very useful in the sequel.

Throughout the paper the letter $E$ will denote any ordinary sequence $E=\left(\varepsilon_{n}\right)$ (integers $n \geq 0$ ) with $0<\varepsilon_{n} \leq 1$ and $\varepsilon_{n} \rightarrow 0$ as $n \rightarrow \infty$. Such a sequence will be termed a fundamental sequence. With this in mind, the following compactness result holds. 
Theorem 1. Any bounded sequence $\left(u_{\varepsilon}\right)_{\varepsilon \in E}$ in $L^{p}(Q \times \Omega)$ (where $E$ is a fundamental sequence and $1<p<\infty)$ admits a subsequence which is weakly stochastically two-scale convergent in $L^{p}(Q \times \Omega)$.

Since the space $L^{p}(\Omega)$ may be not separable, the proof of the above theorem relies on the following result whose proof can be found in [26].

Proposition 4 ([26, Proposition 3.2]). Let $X$ be a subspace (not necessarily closed) of a reflexive Banach space $Y$ and let $f_{n}: X \rightarrow \mathbb{C}$ be a sequence of linear functionals (not necessarily continuous). Assume there exists a constant $c>0$ such that

$$
\limsup _{n}\left|f_{n}(x)\right| \leq c\|x\| \text { for all } x \in X \text {. }
$$

where $\|\cdot\|$ denotes the norm in $Y$. Then there exist a subsequence $\left(f_{n_{k}}\right)_{k}$ of $\left(f_{n}\right)$ and a functional $f \in Y^{\prime}$ such that $\lim _{k} f_{n_{k}}(x)=f(x)$ for all $x \in X$.

Proof of Theorem 1. Let $Y=L^{p^{\prime}}\left(Q \times \Omega ; L_{\text {per }}^{p^{\prime}}(Y)\right), X=\mathcal{C}_{0}^{\infty}(Q) \otimes \mathcal{C}^{\infty}(\Omega) \otimes \mathcal{C}_{\text {per }}(Y)$. Let us define the mapping $L_{\varepsilon}$ by

$$
L_{\varepsilon}(f)=\int_{Q \times \Omega} u_{\varepsilon} f^{\varepsilon} d x d \mu \quad\left(f \in \mathcal{C}_{0}^{\infty}(Q) \otimes \mathcal{C}^{\infty}(\Omega) \otimes \mathcal{C}_{\text {per }}(Y)\right)
$$

where $f^{\varepsilon}(x, \omega)=f\left(x, T\left(x / \varepsilon_{1}\right) \omega, x / \varepsilon_{2}\right)$ for $(x, \omega) \in Q \times \Omega$. Then

$$
\limsup _{\varepsilon}\left|L_{\varepsilon}(f)\right| \leq c\|f\|_{L^{p^{\prime}}(Q \times \Omega \times Y)} \text { for all } f \in X .
$$

Indeed one has the inequality $\left|L_{\varepsilon}(f)\right| \leq c\left\|f^{\varepsilon}\right\|_{L^{p^{\prime}}(Q \times \Omega)}$ and thus, as $\varepsilon \rightarrow 0$, $\left\|f^{\varepsilon}\right\|_{L^{p^{\prime}}(Q \times \Omega)} \rightarrow\|f\|_{L^{p^{\prime}}(Q \times \Omega \times Y)}$ (see (3.5)). We deduce from the Proposition 4 the existence of a subsequence $E^{\prime}$ of $E$ and of a unique $u_{0} \in L^{p}\left(Q \times \Omega ; L_{\mathrm{per}}^{p}(Y)\right)$ such that

$$
\int_{Q \times \Omega} u_{\varepsilon} f^{\varepsilon} d x d \mu \rightarrow \iint_{Q \times \Omega \times Y} u_{0}(x, \omega, y) f(x, \omega, y) d x d \mu d y
$$

for all $f \in \mathcal{C}_{0}^{\infty}(Q) \otimes \mathcal{C}^{\infty}(\Omega) \otimes \mathcal{C}_{\text {per }}(Y)$, whence the theorem.

The next compactness result which is the corner-stone of the homogenization process requires a preliminary lemma.

Lemma 1. Let $\left(u_{\varepsilon}\right)_{\varepsilon>0}$ be a sequence in $L^{p}(Q \times \Omega)(1<p<\infty)$ which stochastically weakly two-scale converges towards $u_{0} \in L^{p}\left(Q \times \Omega ; L_{\mathrm{per}}^{p}(Y)\right)$. Let the sequence $\left(v_{\varepsilon}\right)_{\varepsilon>0}$ be defined by

$$
v_{\varepsilon}(x, \omega)=\int_{B_{r}} u_{\varepsilon}\left(x+\varepsilon_{2} \rho, \omega\right) d \rho \quad((x, \omega) \in Q \times \Omega),
$$

$B_{r}$ being the open ball of $\mathbb{R}^{N}$ centered at 0 and of radius $r>0$. Then, as $\varepsilon \rightarrow 0$,

$$
v_{\varepsilon} \rightarrow v_{0} \text { stoch. in } L^{p}(Q \times \Omega) \text {-weak } 2 s
$$

where $v_{0}$ is defined by $v_{0}(x, \omega, y)=\int_{B_{r}} u_{0}(x, \omega, y+\rho) d \rho$ for $(x, \omega, y) \in Q \times \Omega \times \mathbb{R}^{N}$.

Remark 2. (1) Note that in Lemma 1 , the sequence $\left(v_{\varepsilon}\right)_{\varepsilon>0}$ is well-defined since, as $u_{\varepsilon} \in L^{p}(Q \times \Omega)=L^{p}\left(\Omega ; L^{p}(Q)\right)$, the function $u_{\varepsilon}$ may be extended to an element $\widetilde{u}_{\varepsilon}$ of $L^{p}\left(\Omega ; L_{\text {loc }}^{p}\left(\mathbb{R}^{N}\right)\right)$. (2) Assume that the hypothesis of Lemma 1 holds. Then as $\varepsilon \rightarrow 0$ we have

$$
\frac{1}{\left|B_{\varepsilon_{2} r}\right|} \int_{B_{\varepsilon_{2} r}} u_{\varepsilon}(x+\rho, \omega) d \rho \rightarrow \frac{1}{\left|B_{r}\right|} v_{0} \text { stoch. in } L^{p}(Q \times \Omega) \text {-weak 2s. }
$$


The above convergence result will be of particular interest in the next result.

Proof of Lemma 1. Let $\varphi \in \mathcal{C}_{0}^{\infty}(Q), f \in \mathcal{C}^{\infty}(\Omega)$ and $g \in \mathcal{C}_{\text {per }}(Y)$. One has

$$
\begin{aligned}
& \iint_{Q \times \Omega}\left(\int_{B_{r}} u_{\varepsilon}\left(x+\varepsilon_{2} \rho, \omega\right) d \rho\right) \varphi(x) f\left(T\left(\frac{x}{\varepsilon_{1}}\right) \omega\right) g\left(\frac{x}{\varepsilon_{2}}\right) d x d \mu \\
= & \iint_{B_{r}}\left(\iint_{Q \times \Omega} u_{\varepsilon}\left(x+\varepsilon_{2} \rho, \omega\right) \varphi(x) f\left(T\left(\frac{x}{\varepsilon_{1}}\right) \omega\right) g\left(\frac{x}{\varepsilon_{2}}\right) d x d \mu\right) d \rho .
\end{aligned}
$$

In view of the Lebesgue dominated convergence theorem, (3.6) will be checked as soon as we show that for each fixed $\rho \in \mathbb{R}^{N}$ one has, as $E \ni \varepsilon \rightarrow 0$,

$$
\begin{aligned}
& \iint_{Q \times \Omega} u_{\varepsilon}\left(x+\varepsilon_{2} \rho, \omega\right) \varphi(x) f\left(T\left(\frac{x}{\varepsilon_{1}}\right) \omega\right) g\left(\frac{x}{\varepsilon_{2}}\right) d x d \mu \\
\rightarrow & \iint_{Q \times \Omega} \int_{Y}\left(\tau_{-\rho} u_{0}\right)(x, \omega, y) \varphi(x) f(\omega) g(y) d y d x d \mu .
\end{aligned}
$$

where the function $\tau_{-\rho} u_{0}$ is define by $\left(\tau_{-\rho} u_{0}\right)(x, \omega, y)=u_{0}(x, \omega, y+\rho)$. So let $a \in \mathbb{R}^{N}$ and let $\varphi, f$ and $g$ be as above; then

$$
\begin{aligned}
& \iint_{Q \times \Omega} u_{\varepsilon}\left(x-\varepsilon_{2} a, \omega\right) \varphi(x) f\left(T\left(\frac{x}{\varepsilon_{1}}\right) \omega\right) g\left(\frac{x}{\varepsilon_{2}}\right) d x d \mu= \\
& =\iint_{\left(Q-\varepsilon_{2} a\right) \times \Omega} u_{\varepsilon}(x, \omega) \varphi\left(x+\varepsilon_{2} a\right) f\left(T\left(\frac{x}{\varepsilon_{1}}+\frac{\varepsilon_{2}}{\varepsilon_{1}} a\right) \omega\right) g\left(\frac{x}{\varepsilon_{2}}+a\right) d x d \mu \\
& =\iint_{Q \times \Omega} u_{\varepsilon}(x, \omega) \varphi\left(x+\varepsilon_{2} a\right) f\left(T\left(\frac{x}{\varepsilon_{1}}+\frac{\varepsilon_{2}}{\varepsilon_{1}} a\right) \omega\right) g\left(\frac{x}{\varepsilon_{2}}+a\right) d x d \mu \\
& \quad-\iint_{\left(Q \backslash\left(Q-\varepsilon_{2} a\right)\right) \times \Omega} u_{\varepsilon}(x, \omega) \varphi\left(x+\varepsilon_{2} a\right) f\left(T\left(\frac{x}{\varepsilon_{1}}+\frac{\varepsilon_{2}}{\varepsilon_{1}} a\right) \omega\right) g\left(\frac{x}{\varepsilon_{2}}+a\right) d x d \mu \\
& \quad+\iint_{\left(\left(Q-\varepsilon_{2} a\right) \backslash Q\right) \times \Omega} u_{\varepsilon}(x, \omega) \varphi\left(x+\varepsilon_{2} a\right) f\left(T\left(\frac{x}{\varepsilon_{1}}+\frac{\varepsilon_{2}}{\varepsilon_{1}} a\right) \omega\right) g\left(\frac{x}{\varepsilon_{2}}+a\right) d x d \mu \\
& =(I)-(I I)+(I I I) .
\end{aligned}
$$

As for $(I)$ we have

$$
\begin{aligned}
(I)= & \int_{Q \times \Omega} u_{\varepsilon}(x, \omega) \varphi(x) f\left(T\left(\frac{x}{\varepsilon_{1}}+\frac{\varepsilon_{2}}{\varepsilon_{1}} a\right) \omega\right)\left(\tau_{-a} g\right)\left(\frac{x}{\varepsilon_{2}}\right) d x d \mu \\
& +\int_{Q \times \Omega} u_{\varepsilon}(x, \omega)\left[\varphi\left(x+\varepsilon_{2} a\right)-\varphi(x)\right] f\left(T\left(\frac{x}{\varepsilon_{1}}+\frac{\varepsilon_{2}}{\varepsilon_{1}} a\right) \omega\right)\left(\tau_{-a} g\right)\left(\frac{x}{\varepsilon_{2}}\right) d x d \mu \\
= & \left(I_{1}\right)+\left(I_{2}\right) .
\end{aligned}
$$

But

$$
\begin{aligned}
\left(I_{1}\right)= & \iint_{Q \times \Omega} u_{\varepsilon}(x, \omega) \varphi(x) f\left(T\left(\frac{x}{\varepsilon_{1}}\right) \omega\right)(\tau-a g)\left(\frac{x}{\varepsilon_{2}}\right) d x d \mu \\
& +\iint_{Q \times \Omega} u_{\varepsilon}(x, \omega) \varphi(x)\left(\tau_{-a} g\right)\left(\frac{x}{\varepsilon_{2}}\right)\left[f\left(T\left(\frac{x}{\varepsilon_{1}}+\frac{\varepsilon_{2}}{\varepsilon_{1}} a\right) \omega\right)-f\left(T\left(\frac{x}{\varepsilon_{1}}\right) \omega\right)\right] d x d \mu \\
= & \left(I_{1}^{\prime}\right)+\left(I_{2}^{\prime}\right) .
\end{aligned}
$$

As for $\left(I_{1}^{\prime}\right)$, we have

$$
\left(I_{1}^{\prime}\right) \rightarrow \iint_{Q \times \Omega} \int_{Y} u_{0}(x, \omega, y) \varphi(x) f(\omega)\left(\tau_{-a} g\right)(y) d y d x d \mu \text { as } E \ni \varepsilon \rightarrow 0 .
$$


But

$$
\begin{aligned}
\int_{Y} u_{0}(x, \omega, y)\left(\tau_{-a} g\right)(y) d y & =M\left(u_{0}(x, \omega, \cdot)\left(\tau_{-a} g\right)\right) \\
& =M\left(\tau_{-a}\left[\tau_{a} u_{0}(x, \omega, \cdot) g\right]\right) \\
& =M\left(\tau_{a} u_{0}(x, \omega, \cdot) g\right) \\
& =\int_{Y} \tau_{a} u_{0}(x, \omega, y) g(y) d y
\end{aligned}
$$

where $M$ is the usual mean value in $\mathcal{C}_{\text {per }}(Y)$. For $\left(I_{2}^{\prime}\right)$ we have

$$
\leq \mid\left\|u_{\varepsilon}\right\|_{L^{p}(Q \times \Omega)}\|\varphi\|_{\infty}\|g\|_{\infty}\left(\iint_{Q \times \Omega}\left|f\left(T\left(\frac{x}{\varepsilon_{1}}+\frac{\varepsilon_{2}}{\varepsilon_{1}} a\right) \omega\right)-f\left(T\left(\frac{x}{\varepsilon_{1}}\right) \omega\right)\right|^{p^{\prime}} d x d \mu\right)^{1 / p^{\prime}} .
$$

But

$$
\begin{aligned}
& \iint_{Q \times \Omega}\left|f\left(T\left(\frac{x}{\varepsilon_{1}}+\frac{\varepsilon_{2}}{\varepsilon_{1}} a\right) \omega\right)-f\left(T\left(\frac{x}{\varepsilon_{1}}\right) \omega\right)\right|^{p^{\prime}} d x d \mu \\
= & \iint_{Q \times \Omega}\left|\left(U\left(\frac{x}{\varepsilon_{1}}+\frac{\varepsilon_{2}}{\varepsilon_{1}} a\right) f\right)(\omega)-\left(U\left(\frac{x}{\varepsilon_{1}}\right) f\right)(\omega)\right|^{p^{\prime}} d x d \mu .
\end{aligned}
$$

Since the group $U(x)$ is strongly continuous in $L^{p^{\prime}}(\Omega)$ (see Section 2) we get immediately, using the Lebesgue dominated convergence theorem, that

$$
\iint_{Q \times \Omega}\left|f\left(T\left(\frac{x}{\varepsilon_{1}}+\frac{\varepsilon_{2}}{\varepsilon_{1}} a\right) \omega\right)-f\left(T\left(\frac{x}{\varepsilon_{1}}\right) \omega\right)\right|^{p^{\prime}} d x d \mu \rightarrow 0 \text { as } \varepsilon \rightarrow 0 .
$$

Thus $\left(I_{2}^{\prime}\right) \rightarrow 0$ as $E \ni \varepsilon \rightarrow 0$. Finally since the sequence $\left(u_{\varepsilon}\right)_{\varepsilon \in E}$ is bounded in $L^{p}(Q \times \Omega)$ and as $p>1$, this sequence is uniformly integrable in $L^{1}(Q \times \Omega)$. Therefore the inequality

$$
\begin{aligned}
& \iint_{\left(\left(Q-\varepsilon_{2} a\right) \Delta Q\right) \times \Omega}\left|u_{\varepsilon}(x, \omega)\right|\left|\varphi\left(x+\varepsilon_{2} a\right)\right|\left|f\left(T\left(\frac{x}{\varepsilon_{1}}+\frac{\varepsilon_{2}}{\varepsilon_{1}} a\right) \omega\right)\right|\left|g\left(\frac{x}{\varepsilon_{2}}+a\right)\right| d x d \mu \\
\leq & \|\varphi\|_{\infty}\|f\|_{\infty}\|g\|_{\infty} \iint_{\left(\left(Q-\varepsilon_{2} a\right) \Delta Q\right) \times \Omega}\left|u_{\varepsilon}(x, \omega)\right| d x d \mu
\end{aligned}
$$

yields that $(I I)$ and $(I I I)$ go towards 0 as $E \ni \varepsilon \rightarrow 0$; here the symbol $\Delta$ between the sets $\left(Q-\varepsilon_{2} a\right)$ and $Q$ denotes the symmetric difference between these two sets. Whence the lemma.

We are now able to state and prove the next compactness result. It will be of capital interest in the next section.

Theorem 2. Let $1<p<\infty$. Let $X$ be a norm closed convex subset of $W^{1, p}(Q)$, $Q$ being an open bounded subset of $\mathbb{R}^{N}$. Assume $\left(u_{\varepsilon}\right)_{\varepsilon \in E}$ is a sequence in $L^{p}(Q \times \Omega)$ such that:

(i) $u_{\varepsilon}(\cdot, \omega) \in X$ for all $\varepsilon \in E$ and for $\mu$-a.e. $\omega \in \Omega$;

(ii) $\left(u_{\varepsilon}\right)_{\varepsilon \in E}$ is bounded in $L^{p}\left(\Omega ; W^{1, p}(Q)\right)$.

Then there exist $u_{0} \in W^{1, p}\left(Q ; I_{n v}^{p}(\Omega)\right), u_{1} \in L^{p}\left(Q ; \mathcal{W}^{1, p}(\Omega)\right), u_{2} \in L^{p}(Q \times$ $\left.\Omega ; W_{\#}^{1, p}(Y)\right)$ and a subsequence $E^{\prime}$ from $E$ such that

(iii) $u_{0}(\cdot, \omega) \in X$ for $\mu$-a.e. $\omega \in \Omega$ and, as $E^{\prime} \ni \varepsilon \rightarrow 0$, 
(iv) $u_{\varepsilon} \rightarrow u_{0}$ stoch. in $L^{p}(Q \times \Omega)$-weak (see (3.3))

(v) $D u_{\varepsilon} \rightarrow D u_{0}+\bar{D}_{\omega} u_{1}+D_{y} u_{2}$ stoch. in $L^{p}(Q \times \Omega)^{N}$-weak $2 s$.

Proof. By Theorem 1 there exists a subsequence $E^{\prime}$ from $E$, a function $u_{0} \in L^{p}(Q \times$ $\left.\Omega ; L_{\text {per }}^{p}(Y)\right)$ and a vector function $\mathbf{v}=\left(v_{i}\right)_{1 \leq i \leq N} \in L^{p}\left(Q \times \Omega ; L_{\text {per }}^{p}(Y)\right)^{N}$ such that, as $E^{\prime} \ni \varepsilon \rightarrow 0$, we have $u_{\varepsilon} \rightarrow u_{0}$ stoch. in $L^{p}(Q \times \Omega)$-weak 2s and

$$
D u_{\varepsilon} \rightarrow \mathbf{v} \text { stoch. in } L^{p}(Q \times \Omega)^{N} \text {-weak } 2 \text { s. }
$$

We must check that: $(1)(*) u_{0}$ does not depend upon $y$, that is $\bar{D}_{y} u_{0}=0,(* *)$ $u_{0}(x, \cdot) \in I_{n v}^{p}(\Omega)$, that is $D_{\omega} u_{0}(x, \cdot)=0$ or equivalently $\int_{\Omega} u_{0}(x, \cdot) D_{i, p} \varphi d \mu=$ $0 \forall \varphi \in \mathcal{C}^{\infty}(\Omega)$ and $(* * *) u_{0} \in W^{1, p}\left(Q ; I_{n v}^{p}(\Omega)\right) ;(2) u_{0}(\cdot, \omega) \in X$ for $\mu$-a.e. $\omega \in \Omega$; (3) There exist two functions $u_{1} \in L^{p}\left(Q ; \mathcal{W}^{1, p}(\Omega)\right)$ and $u_{2} \in L^{p}\left(Q \times \Omega ; W_{\#}^{1, p}(Y)\right)$ such that $\mathbf{v}=D u_{0}+\bar{D}_{\omega} u_{1}+D_{y} u_{2}$.

Let us first check (1). $(*)$ Let $\Phi_{\varepsilon}(x, \omega)=\varepsilon_{2} \varphi(x) f\left(T\left(x / \varepsilon_{1}\right) \omega\right) g\left(x / \varepsilon_{2}\right)$ for $(x, \omega) \in$ $Q \times \Omega$, where $\varphi \in \mathcal{C}_{0}^{\infty}(Q), f \in \mathcal{C}^{\infty}(\Omega)$ and $g \in \mathcal{D}_{\text {per }}(Y)=\mathcal{C}_{\text {per }}(Y) \cap \mathcal{C}^{\infty}\left(\mathbb{R}^{N}\right)$. Then

$$
\begin{aligned}
\iint_{Q \times \Omega} \frac{\partial u_{\varepsilon}}{\partial x_{i}} \Phi_{\varepsilon} d x d \mu= & -\iint_{Q \times \Omega} \varepsilon_{2} u_{\varepsilon} f^{\varepsilon} g^{\varepsilon} \frac{\partial \varphi}{\partial x_{i}} d x d \mu-\iint_{Q \times \Omega} u_{\varepsilon} \varphi f^{\varepsilon}\left(D_{y_{i}} g\right)^{\varepsilon} d x d \mu \\
& -\iint_{Q \times \Omega} \frac{\varepsilon_{2}}{\varepsilon_{1}} u_{\varepsilon} \varphi g^{\varepsilon}\left(D_{i, \omega} f\right)^{\varepsilon} d x d \mu
\end{aligned}
$$

where $D_{y_{i}} g=\partial g / \partial y_{i}, f^{\varepsilon}(x, \omega)=f\left(T\left(x / \varepsilon_{1}\right) \omega\right)$ and $g^{\varepsilon}(x)=g\left(x / \varepsilon_{2}\right)$. Letting $E^{\prime} \ni \varepsilon \rightarrow 0$ we get

$$
\iiint_{Q \times \Omega \times Y} u_{0} \varphi D_{y_{i}} g f d x d \mu d y=0
$$

hence $\int_{Y} u_{0}(x, \omega, \cdot) D_{y_{i}} g d y=0$ for all $g \in \mathcal{D}_{\text {per }}(Y)$ and all $1 \leq i \leq N$, which means that $u_{0}$ does not depend on $y$.

(**) Let $\Phi_{\varepsilon}(x, \omega)=\varepsilon_{1} \varphi(x) f\left(T\left(x / \varepsilon_{1}\right) \omega\right)$ for $(x, \omega) \in Q \times \Omega$ where $\varphi \in \mathcal{C}_{0}^{\infty}(Q)$ and $f \in \mathcal{C}^{\infty}(\Omega)$. Then proceeding as above we get $\int_{\Omega} u_{0}(x, \cdot) D_{i, \omega} f d \mu=0$ for all $1 \leq i \leq N$ and all $f \in \mathcal{C}^{\infty}(\Omega)$, which is equivalent to say that $u_{0}(x, \cdot) \in I_{n v}^{p}(\Omega)$ for a.e. $x \in Q$ (use property (P2) in Section 2).

$(* * *)$ Hypothesis (ii) implies that the sequence $\left(u_{\varepsilon}\right)_{\varepsilon \in E^{\prime}}$ is bounded in $W^{1, p}\left(Q ; L^{p}(\Omega)\right)$, which yields the existence of a subsequence of $E^{\prime}$ not relabeled and of a function $u \in W^{1, p}\left(Q ; L^{p}(\Omega)\right)$ such that $u_{\varepsilon} \rightarrow u$ in $W^{1, p}\left(Q ; L^{p}(\Omega)\right)$-weak as $E^{\prime} \ni \varepsilon \rightarrow 0$. In particular $\int_{\Omega} u_{\varepsilon}(\cdot, \omega) \psi(\omega) d \mu \rightarrow \int_{\Omega} u(\cdot, \omega) \psi(\omega) d \mu$ in $L^{1}(Q)$-weak for all $\psi \in I_{n v}^{p^{\prime}}(\Omega)$. Therefore using [10] (see in particular Lemma 3.6 therein) we get at once $u_{0} \in$ $W^{1, p}\left(Q ; L^{p}(\Omega)\right)$, so that $u_{0} \in W^{1, p}\left(Q ; I_{n v}^{p}(\Omega)\right)$.

As for (2), repeating the proof of [part (iii) of ] [10, Theorem 3.7 (b)] we are immediately led to (2). It remains to check (3). We begin by deriving first the existence of $u_{2} \in L^{p}\left(Q \times \Omega ; W_{\#}^{1, p}(Y)\right)$. For that purpose, let $r>0$ be freely fixed. Let $B_{\varepsilon_{2} r}$ denote the open ball in $\mathbb{R}^{N}$ centered at the origin and of radius $\varepsilon_{2} r$. By 
the equalities

$$
\begin{aligned}
& \frac{1}{\varepsilon_{2}}\left(u_{\varepsilon}(x, \omega)-\frac{1}{\left|B_{\varepsilon_{2} r}\right|} \int_{B_{\varepsilon_{2} r}} u_{\varepsilon}(x+\rho, \omega) d \rho\right) \\
= & \frac{1}{\varepsilon_{2}} \frac{1}{\left|B_{\varepsilon_{2} r}\right|} \int_{B_{\varepsilon_{2} r}}\left(u_{\varepsilon}(x, \omega)-u_{\varepsilon}(x+\rho, \omega)\right) d \rho \\
= & \frac{1}{\varepsilon_{2}} \frac{1}{\left|B_{r}\right|} \int_{B_{r}}\left(u_{\varepsilon}(x, \omega)-u_{\varepsilon}\left(x+\varepsilon_{2} \rho, \omega\right)\right) d \rho \\
= & -\frac{1}{\left|B_{r}\right|} \int_{B_{r}} d \rho \int_{0}^{1} D u_{\varepsilon}\left(x+t \varepsilon_{2} \rho, \omega\right) \cdot \rho d t
\end{aligned}
$$

where the dot denotes the usual Euclidean inner product in $\mathbb{R}^{N}$, we deduce from the boundedness of $\left(u_{\varepsilon}\right)_{\varepsilon \in E^{\prime}}$ in $L^{p}\left(\Omega ; W^{1, p}(Q)\right)$ that the sequence $\left(z_{\varepsilon}^{r}\right)_{\varepsilon \in E^{\prime}}$ defined by

$$
z_{\varepsilon}^{r}(x, \omega)=\frac{1}{\varepsilon_{2}}\left(u_{\varepsilon}(x, \omega)-\frac{1}{\left|B_{\varepsilon_{2} r}\right|} \int_{B_{\varepsilon_{2} r}} u_{\varepsilon}(x+\rho, \omega) d \rho\right) \quad\left((x, \omega) \in Q \times \Omega, \varepsilon \in E^{\prime}\right)
$$

is bounded in $L^{p}(Q \times \Omega)$. It is important to note that in general the function $z_{\varepsilon}^{r}$ is well defined since $u_{\varepsilon}$ and $D u_{\varepsilon}$ can be naturally extended off $Q$ as elements of $L^{p}\left(\Omega ; L_{\text {loc }}^{p}\left(\mathbb{R}^{N}\right)\right)$ and $L^{p}\left(\Omega ; L_{\text {loc }}^{p}\left(\mathbb{R}^{N}\right)^{N}\right)$, respectively. Thus, it results once more from Theorem 1 the existence of a subsequence from $E^{\prime}$ not relabeled and of a function $z_{r}$ in $L^{p}\left(Q \times \Omega ; L_{\mathrm{per}}^{p}(Y)\right)$ such that, as $E^{\prime} \ni \varepsilon \rightarrow 0$

$$
z_{\varepsilon}^{r} \rightarrow z_{r} \text { stoch. in } L^{p}(Q \times \Omega) \text {-weak } 2 \text { s. }
$$

As $\left(z_{\varepsilon}^{r}\right)_{\varepsilon \in E^{\prime}}$ is bounded in $L^{p}(Q \times \Omega)$ we have (since $\varepsilon_{2}, \varepsilon_{2} / \varepsilon_{1} \rightarrow 0$ as $E^{\prime} \ni \varepsilon \rightarrow 0$ ) that

$$
\varepsilon_{2} z_{\varepsilon}^{r} \rightarrow 0 \text { in } L^{p}(Q \times \Omega) \text { and } \frac{\varepsilon_{2}}{\varepsilon_{1}} z_{\varepsilon}^{r} \rightarrow 0 \text { in } L^{p}(Q \times \Omega) \text { as } E^{\prime} \ni \varepsilon \rightarrow 0 .
$$

Now, for $\varphi \in \mathcal{C}_{0}^{\infty}(Q), f \in \mathcal{D}(\Omega)$ and $g \in \mathcal{D}_{\text {per }}(Y)$ we have

$$
\begin{aligned}
& \iint_{Q \times \Omega}\left(\frac{\partial u_{\varepsilon}}{\partial x_{i}}(x, \omega)-\frac{1}{\left|B_{\varepsilon_{2} r}\right|} \int_{B_{\varepsilon_{2} r}} \frac{\partial u_{\varepsilon}}{\partial x_{i}}(x+\rho, \omega) d \rho\right) \varphi(x) f\left(T\left(\frac{x}{\varepsilon_{1}}\right) \omega\right) g\left(\frac{x}{\varepsilon_{2}}\right) d x d \mu \\
& =-\iint_{Q \times \Omega} \varepsilon_{2} z_{\varepsilon}^{r}(x, \omega) f\left(T\left(\frac{x}{\varepsilon_{1}}\right) \omega\right) g\left(\frac{x}{\varepsilon_{2}}\right) \frac{\partial \varphi}{\partial x_{i}}(x) d x d \mu \\
& -\iint_{Q \times \Omega} \frac{\varepsilon_{2}}{\varepsilon_{1}} z_{\varepsilon}^{r}(x, \omega) \varphi(x) g\left(\frac{x}{\varepsilon_{2}}\right)\left(D_{i, \omega} f\right)\left(T\left(\frac{x}{\varepsilon_{1}}\right) \omega\right) d x d \mu \\
& \quad-\iint_{Q \times \Omega} z_{\varepsilon}^{r}(x, \omega) \varphi(x) f\left(T\left(\frac{x}{\varepsilon_{1}}\right) \omega\right) \frac{\partial g}{\partial y_{i}}\left(\frac{x}{\varepsilon_{2}}\right) d x d \mu .
\end{aligned}
$$

Passing to the limit in (3.11) (as $E^{\prime} \ni \varepsilon \rightarrow 0$ ) using conjointly (3.8), (3.9), (3.10) and Remark 2 (see (3.7) therein) one gets

$$
\begin{aligned}
& \iiint_{Q \times \Omega \times Y}\left(v_{i}(x, \omega, y)-\frac{1}{\left|B_{r}\right|} \int_{B_{r}} v_{i}(x, \omega, y+\rho) d \rho\right) \varphi(x) f(\omega) g(y) d x d \mu d y \\
& \quad=-\iiint_{Q \times \Omega \times Y} z_{r}(x, \omega, y) \varphi(x) f(\omega) \frac{\partial g}{\partial y_{i}}(y) d x d \mu d y .
\end{aligned}
$$

Since $\varphi, f$ and $g$ are arbitrary, we arrive at

$$
\frac{\partial z_{r}}{\partial y_{i}}(x, \omega, y)=v_{i}(x, \omega, y)-\frac{1}{\left|B_{r}\right|} \int_{B_{r}} v_{i}(x, \omega, y+\rho) d \rho
$$

a.e. in $x \in Q, \omega \in \Omega$ and $y \in \mathbb{R}^{N}$. Set $f_{r}(x, \omega, y)=z_{r}(x, \omega, y)-M\left(z_{r}(x, \omega, \cdot)\right)$ for a.e. $(x, \omega, y) \in Q \times \Omega \times \mathbb{R}^{N}$, where we recall that $M\left(z_{r}(x, \omega, \cdot)\right)=\int_{Y} z_{r}(x, \omega, y) d y$; 
then $M\left(f_{r}(x, \omega, \cdot)\right)=0$ and moreover $D_{y} f_{r}(x, \omega, \cdot)=D_{y} z_{r}(x, \omega, \cdot)$ for a.e. $(x, \omega) \in$ $Q \times \Omega$, so that $f_{r} \in L^{p}\left(Q \times \Omega ; L_{\#}^{p}(Y)\right)\left(L_{\#}^{p}(Y)\right.$ the space of those $u \in L_{\mathrm{per}}^{p}(Y)$ with $M(u)=0)$ with $\partial f_{r} / \partial y_{i} \in L^{p}\left(Q \times \Omega ; L_{\text {per }}^{p}(Y)\right)$ for all $1 \leq i \leq N$, that is, $f_{r} \in L^{p}\left(Q \times \Omega ; W_{\#}^{1, p}(Y)\right)$. Moreover we have

$$
\frac{\partial f_{r}}{\partial y_{i}}(x, \omega, \cdot)=v_{i}(x, \omega, \cdot)-\frac{1}{\left|B_{r}\right|} \int_{B_{r}} v_{i}(x, \omega, \cdot+\rho) d \rho(1 \leq i \leq N) .
$$

As the function $v_{i}$ is periodic in its third argument $y$, we have that, when $r \rightarrow+\infty$, the right-hand side of the above equality goes towards $v_{i}(x, \omega, \cdot)-M\left(v_{i}(x, \omega, \cdot)\right)$, whence the existence of a unique function $u_{2}(x, \omega, \cdot) \in W_{\#}^{1, p}(Y)$ such that

$$
D_{y} u_{2}(x, \omega, \cdot)=\mathbf{v}(x, \omega, \cdot)-M(\mathbf{v}(x, \omega, \cdot)) d x \times d \mu \text {-a.e. }(x, \omega) \in Q \times \Omega,
$$

hence the existence of a unique $u_{2}: Q \times \Omega \rightarrow W_{\#}^{1, p}(Y),(x, \omega) \mapsto u_{2}(x, \omega, \cdot)$, lying in $L^{p}\left(Q \times \Omega ; W_{\#}^{1, p}(Y)\right)$ such that

$$
\mathbf{v}-M(\mathbf{v})=D_{y} u_{2}
$$

Let us finally derive the existence of $u_{1}$. Let $\Phi_{\varepsilon}(x, \omega)=\varphi(x) \Psi\left(T\left(x / \varepsilon_{1}\right) \omega\right)((x, \omega) \in$ $Q \times \Omega)$ with $\varphi \in \mathcal{C}_{0}^{\infty}(Q)$ and $\Psi=\left(\psi_{j}\right)_{1 \leq j \leq N} \in \mathcal{V}_{\text {div }}$ (i.e. $\operatorname{div}_{\omega, p^{\prime}} \Psi=0$ ). Clearly

$$
\sum_{j=1}^{N} \iint_{Q \times \Omega} \frac{\partial u_{\varepsilon}}{\partial x_{j}} \varphi \psi_{j}^{\varepsilon} d x d \mu=-\sum_{j=1}^{N} \iint_{Q \times \Omega} u_{\varepsilon} \psi_{j}^{\varepsilon} \frac{\partial \varphi}{\partial x_{j}} d x d \mu
$$

where $\psi_{j}^{\varepsilon}(x, \omega)=\psi_{j}\left(T\left(x / \varepsilon_{1}\right) \omega\right)$. The limit passage (when $E^{\prime} \ni \varepsilon \rightarrow 0$ ) yields

$$
\sum_{j=1}^{N} \iiint_{Q \times \Omega \times Y} v_{j} \varphi \psi_{j} d x d \mu d y=-\sum_{j=1}^{N} \iiint_{Q \times \Omega \times Y} u_{0} \psi_{j} \frac{\partial \varphi}{\partial x_{j}} d x d \mu d y
$$

or equivalently,

$$
\iiint_{Q \times \Omega \times Y}\left(\mathbf{v}(x, \omega, y)-D u_{0}(x, \omega)\right) \cdot \Psi(\omega) \varphi(x) d x d \mu d y=0
$$

and so, as $\varphi$ is arbitrarily fixed in $\mathcal{C}_{0}^{\infty}(Q)$,

$$
\iint_{\Omega \times Y}\left(\mathbf{v}(x, \omega, y)-D u_{0}(x, \omega)\right) \cdot \Psi(\omega) d \mu d y=0 \quad \forall \Psi \in \mathcal{V}_{\text {div }} .
$$

This is also equivalent to

$$
\int_{\Omega}\left(M(\mathbf{v}(x, \omega, \cdot))-D u_{0}(x, \omega)\right) \cdot \Psi(\omega) d \mu=0 \text { for } \Psi \in \mathcal{V}_{\text {div }} \text { and for a.e. } x \in Q
$$

Therefore, Proposition 1 provides us with a unique $u_{1}(x, \cdot) \in \mathcal{W}^{1, p}(\Omega)$ such that

$$
M(\mathbf{v}(x, \cdot, \cdot))-D u_{0}(x, \cdot)=\bar{D}_{\omega} u_{1}(x, \cdot) \mu \text {-a.e. } x \in Q,
$$

the above equality being reading as, for a.e. $x \in Q, M(\mathbf{v}(x, \omega, \cdot))-D u_{0}(x, \omega)=$ $\bar{D}_{\omega} u_{1}(x, \omega)$ for $\mu$-a.e. $\omega \in \Omega$. Putting (3.12) and (3.13) together leads at once to $\mathbf{v}=D u_{0}+\bar{D}_{\omega} u_{1}+D_{y} u_{2}$ with $u_{1} \in L^{p}\left(Q ; \mathcal{W}^{1, p}(\Omega)\right)$. 
Remark 3. It is worth noting that in [33], Telega and Bielski stated the above convergence method (see Definition 1) and have given some essential properties, but unfortunately they provided no proof to their results. So our work seems to be the first one in which a systematic and rigorous treatment of this notion is carried out.

\section{Application to the homogenization of an integral functional With CONVEX INTEGRAND}

4.1. Setting of the problem. Let $(\Omega, \mathcal{M}, \mu)$ denote a probability space and let $\left\{T(x): x \in \mathbb{R}^{N}\right\}$ denote an $N$-dimensional dynamical system acting on the above probability space. We intend to study the asymptotic behaviour (as $0<\varepsilon \rightarrow 0$ ) of the sequence of solutions to the problems

$$
\min \left\{F_{\varepsilon}(v): v \in L^{p}\left(\Omega ; W_{0}^{1, p}\left(Q ; \mathbb{R}^{n}\right)\right)\right\}
$$

where the functional $F_{\varepsilon}$ is defined on $L^{p}\left(\Omega ; W_{0}^{1, p}\left(Q ; \mathbb{R}^{n}\right)\right)$ by

$$
F_{\varepsilon}(v)=\iint_{Q \times \Omega} f\left(x, T\left(\frac{x}{\varepsilon_{1}}\right) \omega, \frac{x}{\varepsilon_{2}}, D v(x, \omega)\right) d x d \mu\left(v \in L^{p}\left(\Omega ; W_{0}^{1, p}\left(Q ; \mathbb{R}^{n}\right)\right)\right),
$$

$Q$ being a bounded open set in $\mathbb{R}^{N}$ (integers $n, N \geq 1$ ), $D$ denoting the gradient operator in $Q$ with respect to $x, \varepsilon_{1}$ and $\varepsilon_{2}$ being to well-separate scales, and the function $f: \mathbb{R}^{N} \times \Omega \times \mathbb{R}^{N} \times \mathbb{R}^{n N} \rightarrow[0,+\infty)$ is a random integrand, that is, $f$ is $\left(\mathcal{B}_{\mathbb{R}^{N}} \times \mathcal{M} \times \mathcal{B}_{\mathbb{R}^{N}} \times \mathcal{B}_{\mathbb{R}^{n N}} ; \mathcal{B}_{\mathbb{R}}\right)$-measurable $\left(\mathcal{B}_{X}\right.$ denotes the Borel $\sigma$-algebra on the topological space $X$ ) and satisfies the following conditions:

$\left(\mathrm{H}_{1}\right)$ There exist a continuous positive function $\varpi: \mathbb{R} \rightarrow \mathbb{R}_{+} \equiv[0,+\infty)$ with $\varpi(0)=0$, and a function $a \in L_{\text {loc }}^{1}\left(\mathbb{R}_{y}^{N}\right)$ such that

$$
\left|f(x, \omega, y, \lambda)-f\left(x^{\prime}, \omega, y, \lambda\right)\right| \leq \varpi\left(\left|x-x^{\prime}\right|\right)(a(y)+f(x, \omega, y, \lambda))
$$

for all $x, x^{\prime} \in \mathbb{R}^{N}, \lambda \in \mathbb{R}^{n N}$ and for $d \mu \times d x$-almost all $(\omega, y) \in \Omega \times \mathbb{R}^{N}$,

$\left(\mathrm{H}_{2}\right)$ For all $(x, y, \lambda) \in \mathbb{R}^{N} \times \mathbb{R}^{N} \times \mathbb{R}^{n N}$ and for almost all $\omega \in \Omega, f(x, \cdot, y, \lambda)$ is measurable and $f(\cdot, \omega, \cdot, \cdot)$ is continuous,

$\left(\mathrm{H}_{3}\right) f(x, \omega, y, \cdot)$ is strictly convex for $d \mu \times d x$-almost all $(\omega, y) \in \Omega \times \mathbb{R}^{N}$ and for all $x \in \mathbb{R}^{N}$,

$\left(\mathrm{H}_{4}\right)$ There exist three constants $p>1$ and $c_{1}, c_{2}>0$ such that

$$
c_{1}|\lambda|^{p} \leq f(x, \omega, y, \lambda) \leq c_{2}\left(1+|\lambda|^{p}\right)
$$

for all $(x, \lambda) \in \mathbb{R}^{N} \times \mathbb{R}^{n N}$ and for $d \mu \times d x$-almost all $(\omega, y) \in \Omega \times \mathbb{R}^{N}$.

Since the function $f(x, \omega, y, \cdot)$ is convex, it comes from (4.3) (see in particular the right-hand side of the inequality in (4.3)) that

$$
\begin{aligned}
& \left|f(x, \omega, y, \lambda)-f\left(x, \omega, y, \lambda^{\prime}\right)\right| \leq c_{2}\left(1+|\lambda|^{p-1}+\left|\lambda^{\prime}\right|^{p-1}\right)\left|\lambda-\lambda^{\prime}\right| \\
& \text { for all } x \in \mathbb{R}^{N}, \lambda, \lambda^{\prime} \in \mathbb{R}^{n N} \text { for } d \mu \times d x \text {-almost all }(\omega, y) \in \Omega \times \mathbb{R}^{N} .
\end{aligned}
$$

Consequently, for any fixed $\varepsilon>0$ and for $v \in L^{p}\left(Q \times \Omega ; \mathbb{R}^{n N}\right)$, the function $(x, \omega) \mapsto$ $f\left(x, T\left(x / \varepsilon_{1}\right) \omega, x / \varepsilon_{2}, v(x, \omega)\right)$ of $Q \times \Omega$ into $\mathbb{R}_{+}$(denoted by $f^{\varepsilon}(\cdot, \cdot, v)$ ), is well defined and lies in $L^{1}(Q \times \Omega)$ (see either [6] or [27]), with

$$
c_{1}\|v\|_{L^{p}(Q \times \Omega)^{n N}}^{p} \leq\left\|f^{\varepsilon}(\cdot, \cdot, v)\right\|_{L^{1}(Q \times \Omega)} \leq c_{2}^{\prime}\left(1+\|v\|_{L^{p}(Q \times \Omega)^{n N}}^{p}\right)
$$


where $c_{2}^{\prime}=c_{2} \max (1,|Q|)$ with $|Q|=\int_{Q} d x$. Therefore, using together the inequality (4.4) (which implies the continuity of the functional $F_{\varepsilon}$ ) with the strict convexity of $F_{\varepsilon}$ (see $\left(\mathrm{H}_{3}\right)$ ) and the left-hand side of the inequality in (4.5) (which means the coercivity of $F_{\varepsilon}$ ), we deduce the existence $[12,19,40]$ of a unique $u_{\varepsilon} \in L^{p}\left(\Omega ; W_{0}^{1, p}\left(Q ; \mathbb{R}^{n}\right)\right)$ solution to the minimization problem

$$
F_{\varepsilon}\left(u_{\varepsilon}\right)=\min _{v \in L^{p}\left(\Omega ; W_{0}^{1, p}\left(Q ; \mathbb{R}^{n}\right)\right)} F_{\varepsilon}(v) .
$$

Our main objective in this section is the study of the asymptotic behaviour (as $0<\varepsilon \rightarrow 0$ ) of the sequence $\left(u_{\varepsilon}\right)_{\varepsilon>0}$ under the periodicity assumption on the function $y \mapsto f(x, \omega, y, \lambda)$ (for fixed $(x, \omega, \lambda))$, that is, the function $f(x, \omega, \cdot, \lambda)$ satisfies the following condition:

$\left(\mathrm{H}_{5}\right)$ For each $k \in \mathbb{Z}^{N}$ we have $f(x, \omega, y+k, \lambda)=f(x, \omega, y, \lambda)$ for all $y \in \mathbb{R}^{N}$.

Under the above assumption $\left(\mathrm{H}_{5}\right)$, the homogenization of functionals $F_{\varepsilon}$ amounts to finding a homogenized functional $F$ such that the sequence of minimizers $u_{\varepsilon}$ converges to a limit $\mathbf{u}$, which turns out to be the minimizer of $F$. Henceforth we shall use hypothesis $\left(\mathrm{H}_{5}\right)$ without any precision.

From a physical point of view, e.g., in elasticity theory, the term $F_{\varepsilon}(v)$ can be viewed as the energy under a deformation $v$ of an elastic body whose microstructures behave randomly in some scales and periodically in the other scales. In the deterministic case where the microstructures have the only periodic behaviour (thus do not depend on the random variable $\omega$ ), the functionals $F_{\varepsilon}$ have attracted the attention of a great number of researchers. We refer, e.g., to $[5,6,11,18,21]$. In the stochastic framework, few results exist in the literature. Indeed in this context, the only results available so far are those associated with functionals of the form $\int_{Q} f(\omega, x / \varepsilon, D v(x)) d x$ where $f$ is a random homogeneous integrand; see, e.g., $[13,14,22,31,32]$. This is justified by the fact that so far only one microscopic scale is considered at once (mostly the deterministic scale in almost all the references cited above, the random scale appearing almost as a parameter). Our choice to consider two separate scales is motivated by physical consideration: a scale can not be both deterministic and random, so we need to deal with at least two different scales. That said, our work seems to be the first one where such a coupled - deterministic and stochastic - environment is considered in the framework of homogenization of functionals of the calculus of variations. Here we choose a convex functional just to simplify the presentation of the paper. The method used here can be applied to non-convex functionals, with some additional assumptions on the integrand $f$ as usual (for instance, the uniform continuity in his last occurrence). It is also important to note that our results generalize those of Braides and Lukkassen [11] where the homogenization problem for the functional $\int_{Q} f\left(x / \varepsilon, x / \varepsilon^{2}, D v(x)\right) d x$ was considered under the assumptions that $f(\cdot, \cdot, \lambda)$ is periodic and $f(y, z, \cdot)$ is convex together with hypothesis $\left(\mathrm{H}_{4}\right)$. Indeed when we consider the particular dynamical system $T(x)$ on $\Omega=\mathbb{T}^{N} \equiv \mathbb{R}^{N} / \mathbb{Z}^{N}$ (the $N$-dimensional torus) defined by $T(x) \omega=x+\omega \bmod \mathbb{Z}^{N}$, then one can view $\Omega$ as the unit cube in $\mathbb{R}^{N}$ with all the pairs of antipodal faces being identified. The Lebesgue measure on $\mathbb{R}^{N}$ induces the Haar measure on $\mathbb{T}^{N}$ which is invariant with respect to the action of $T(x)$ on $\mathbb{T}^{N}$. Moreover $T(x)$ is ergodic, and in this situation, any function on $\Omega$ may be regarded as a periodic function on $\mathbb{R}^{N}$ whose period in each coordinate is 1 , so that in this 
case our integrand $f$ may be viewed as a periodic function with respect to both variables $\omega$ and $y$. Therefore, problem (4.6) is equivalent to the problem

$$
\min _{v \in W_{0}^{1, p}\left(Q ; \mathbb{R}^{n}\right)} \int_{Q} f\left(x, \frac{x}{\varepsilon_{1}}, \frac{x}{\varepsilon_{2}}, D v(x)\right) d x
$$

where the function $f(x, \cdot, \cdot, \lambda)$ is periodic in each of its arguments. We thus obtain by this a generalization of the above-mentioned results by Braides and Lukkassen. It is worth mentioning that the papers $[1,17,34,35,40]$ deal with some interesting problems of stochastic homogenization, but using the monoscale approach. We also mention the paper [25] in which a general deterministic homogenization of convex integral functionals is considered, but still using the monoscale approach.

4.2. Preliminary results. Let $\Psi \in\left(\mathcal{C}^{\infty}(\Omega) \otimes \mathcal{C}\left(\bar{Q} ; \mathcal{C}_{\text {per }}(Y)\right)\right)^{n N}$. The function $(\omega, y) \mapsto f(x, \omega, y, \Psi(x, \omega, y))$ of $\Omega \times \mathbb{R}_{y}^{N}$ into $\mathbb{R}_{+}$, denoted by $f(x, \cdot, \cdot, \Psi(x, \cdot, \cdot))$, lies in $L^{\infty}\left(\Omega \times \mathbb{R}_{y}^{N}\right)$. Whence one can define the function $x \mapsto f(x, \cdot, \cdot, \Psi(x, \cdot, \cdot))$ of $\bar{Q}$ into $L^{\infty}\left(\Omega \times \mathbb{R}_{y}^{N}\right)$ (denoted by $\left.f(\cdot, \cdot, \Psi)\right)$ as element of $\mathcal{C}\left(\bar{Q} ; L^{\infty}\left(\Omega \times \mathbb{R}_{y}^{N}\right)\right)$. Therefore, for fixed $\varepsilon>0$, we define the function $(x, \omega) \mapsto f\left(x, T\left(x / \varepsilon_{1}\right) \omega, x / \varepsilon_{2}, \Psi\left(x, T\left(x / \varepsilon_{1}\right) \omega\right.\right.$, $\left.x / \varepsilon_{2}\right)$ ), denoted by $f^{\varepsilon}\left(\cdot, \cdot, \Psi^{\varepsilon}\right)$, as element of $L^{\infty}(Q \times \Omega)$. Moreover we have, in view of $\left(\mathrm{H}_{5}\right)$,

$$
f(\cdot, \cdot, \Psi) \in \mathcal{C}\left(\bar{Q} ; L^{\infty}\left(\Omega ; L_{\text {per }}^{\infty}(Y)\right) \text { for all } \Psi \in\left[\mathcal{C}^{\infty}(\Omega) \otimes \mathcal{C}\left(\bar{Q} ; \mathcal{C}_{\text {per }}(Y)\right)\right]^{n N} .\right.
$$

We can now state and prove the first preliminary result which will allow us to rigorously set the homogenized problem.

Proposition 5. Let $1<p<\infty$. For any $\Psi \in\left[\mathcal{C}^{\infty}(\Omega) \otimes \mathcal{C}\left(\bar{Q} ; \mathcal{C}_{\text {per }}(Y)\right)\right]^{n N}$ we have

$$
f^{\varepsilon}\left(\cdot, \cdot, \Psi^{\varepsilon}\right) \rightarrow f(\cdot, \cdot, \Psi) \text { stoch. in } L^{1}(Q \times \Omega) \text {-weak } 2 s \text { as } \varepsilon \rightarrow 0 \text {. }
$$

The mapping $\Psi \mapsto f(\cdot, \cdot, \Psi)$ of $\left[\mathcal{C}^{\infty}(\Omega) \otimes \mathcal{C}\left(\bar{Q} ; \mathcal{C}_{\text {per }}(Y)\right)\right]^{n N}$ into $L^{1}(Q \times \Omega \times Y)$ extends by continuity to a mapping still denoted by $f$, of $L^{p}\left(Q \times \Omega ; L_{\mathrm{per}}^{p}(Y)^{n N}\right)$ into $L^{1}(Q \times \Omega \times Y)$ such that

$$
\begin{aligned}
& \|f(\cdot, \cdot, \mathbf{u})-f(\cdot, \cdot, \mathbf{v})\|_{L^{1}(Q \times \Omega \times Y)} \leq \\
& \quad c\left(1+\|\mathbf{u}\|_{L^{p}(Q \times \Omega \times Y)^{n N}}^{p-1}+\|\mathbf{v}\|_{L^{p}(Q \times \Omega \times Y)^{n N}}^{p-1}\right)^{\frac{p-1}{p}}\|\mathbf{u}-\mathbf{v}\|_{L^{p}(Q \times \Omega \times Y)^{n N}}
\end{aligned}
$$

for all $\mathbf{u}, \mathbf{v} \in L^{p}\left(Q \times \Omega ; L_{\mathrm{per}}^{p}(Y)^{n N}\right)$.

Proof. Let $\Psi$ be as above. We have seen a while ago that the function $f(\cdot, \cdot, \Psi)$ lies in $\mathcal{C}\left(\bar{Q} ; L^{\infty}\left(\Omega ; L_{\text {per }}^{p}(Y)\right)\right)$ so that the convergence result $(4.7)$ holds. On the other hand, by the definition of the function $f(\cdot, \cdot, \Psi)$ it is immediate that this function verifies property of the same type as in (4.4), so that arguing as in the proof of [37, Proposition 3.1] we get the remainder of the above proposition.

The next corollary will be of great interest in the proof of the main result of this section.

Corollary 1. Let

$$
\Phi_{\varepsilon}(x, \omega)=\psi_{0}(x, \omega)+\varepsilon_{1} \psi_{1}\left(x, T\left(x / \varepsilon_{1}\right) \omega\right)+\varepsilon_{2} \psi_{2}\left(x, T\left(x / \varepsilon_{1}\right) \omega, x / \varepsilon_{2}\right)
$$

for $(x, \omega) \in Q \times \Omega$, where $\psi_{0} \in\left[\mathcal{C}_{0}^{\infty}(Q) \otimes I_{n v}^{p}(\Omega)\right]^{n}, \psi_{1} \in\left[\mathcal{C}_{0}^{\infty}(Q) \otimes \mathcal{C}^{\infty}(\Omega)\right]^{n}$ and $\psi_{2} \in\left[\mathcal{C}_{0}^{\infty}(Q) \otimes \mathcal{C}^{\infty}(\Omega) \otimes \mathcal{D}_{\#}(Y)\right]^{n}$. Then, as $\varepsilon \rightarrow 0$,

$$
f^{\varepsilon}\left(\cdot, \cdot, D \Phi_{\varepsilon}\right) \rightarrow f\left(\cdot, \cdot, D \psi_{0}+D_{\omega} \psi_{1}+D_{y} \psi_{2}\right) \text { stoch. in } L^{1}(Q \times \Omega) \text {-weak } 2 s .
$$


Proof. As $D \psi_{1}^{\varepsilon}=\left(D \psi_{1}\right)^{\varepsilon}+\frac{1}{\varepsilon_{1}}\left(D_{\omega} \psi_{1}\right)^{\varepsilon}$ (see property (P1) in Section 2), we have that $D \Phi_{\varepsilon}=D \psi_{0}+\left(D_{\omega} \psi_{1}\right)^{\varepsilon}+\left(D_{y} \psi_{2}\right)^{\varepsilon}+\varepsilon_{1}\left(D \psi_{1}\right)^{\varepsilon}+\varepsilon_{2}\left(D \psi_{2}\right)^{\varepsilon}+\varepsilon_{2} / \varepsilon_{1}\left(D_{\omega} \psi_{2}\right)^{\varepsilon}$, and so, by (4.4) (where we have taken there $y=x / \varepsilon_{2}, \lambda=D \psi_{0}+\left(D_{\omega} \psi_{1}\right)^{\varepsilon}+\left(D_{y} \psi_{2}\right)^{\varepsilon}$, $\lambda^{\prime}=D \Phi_{\varepsilon}$ and have also replaced there $\omega$ by $T\left(x / \varepsilon_{1}\right) \omega$, and then after integrating the resulting inequality over $Q \times \Omega$ ) that, as $\varepsilon \rightarrow 0$,

$$
f^{\varepsilon}\left(\cdot, \cdot, D \Phi_{\varepsilon}\right)-f^{\varepsilon}\left(\cdot, \cdot, D \psi_{0}+\left(D_{\omega} \psi_{1}\right)^{\varepsilon}+\left(D_{y} \psi_{2}\right)^{\varepsilon}\right) \rightarrow 0 \text { in } L^{1}(Q \times \Omega) .
$$

Thus by the decomposition (for $v \in L^{\infty}\left(Q \times \Omega ; \mathcal{C}_{\text {per }}(Y)\right)$ )

$$
\begin{aligned}
& \iint_{Q \times \Omega} f^{\varepsilon}\left(\cdot, \cdot, D \Phi_{\varepsilon}\right) v^{\varepsilon} d x d \mu-\iiint_{Q \times \Omega \times Y} f\left(\cdot, \cdot, D \psi_{0}+D_{\omega} \psi_{1}+D_{y} \psi_{2}\right) v d x d \mu d y \\
= & \iint_{Q \times \Omega}\left[f^{\varepsilon}\left(\cdot, \cdot, D \Phi_{\varepsilon}\right)-f^{\varepsilon}\left(\cdot, \cdot, D \psi_{0}+\left(D_{\omega} \psi_{1}\right)^{\varepsilon}+\left(D_{y} \psi_{2}\right)^{\varepsilon}\right)\right] v^{\varepsilon} d x d \mu \\
\quad & +\iint_{Q \times \Omega} f^{\varepsilon}\left(\cdot, \cdot, D \psi_{0}+\left(D_{\omega} \psi_{1}\right)^{\varepsilon}+\left(D_{y} \psi_{2}\right)^{\varepsilon}\right) v^{\varepsilon} d x d \mu \\
\quad & -\iiint_{Q \times \Omega \times Y} f\left(\cdot, \cdot, D \psi_{0}+D_{\omega} \psi_{1}+D_{y} \psi_{2}\right) v d x d \mu d y,
\end{aligned}
$$

the result follows at once by (4.10) and by the convergence result (4.7) in Proposition 5 .

Let $1<p<\infty$. Set

$$
\mathbb{F}_{0}^{1, p}=W_{0}^{1, p}\left(Q ; I_{n v}^{p}(\Omega)\right)^{n} \times L^{p}\left(Q ; \mathcal{W}^{1, p}(\Omega)^{n}\right) \times L^{p}\left(Q \times \Omega ; W_{\#}^{1, p}(Y)^{n}\right)
$$

where $\mathcal{W}^{1, p}(\Omega)^{n}=\left[\mathcal{W}^{1, p}(\Omega)\right]^{n}$ and $W_{\#}^{1, p}(Y)^{n}=\left[W_{\#}^{1, p}(Y)\right]^{n}$. We equip $\mathbb{F}_{0}^{1, p}$ with the norm

$$
\begin{aligned}
\|\mathbf{u}\|_{\mathbb{F}_{0}^{1, p}} & =\left(\left\|u_{0}\right\|_{W_{0}^{1, p}\left(Q ; I_{n v}^{p}(\Omega)\right)^{n}}^{p}+\left\|u_{1}\right\|_{L^{p}\left(Q ; \mathcal{W}^{1, p}(\Omega)^{n}\right)}^{p}+\left\|u_{2}\right\|_{L^{p}\left(Q \times \Omega ; W_{\#}^{1, p}(Y)^{n}\right)}^{p}\right)^{\frac{1}{p}} \\
\text { for } \mathbf{u} & =\left(u_{0}, u_{1}, u_{2}\right) \in \mathbb{F}_{0}^{1, p}
\end{aligned}
$$

where $\left\|u_{0}\right\|_{W_{0}^{1, p}\left(Q ; I_{n v}^{p}(\Omega)\right)^{n}}=\left\|D u_{0}\right\|_{L^{p}(Q \times \Omega)^{n}}$,

$$
\left\|u_{1}\right\|_{L^{p}\left(Q ; \mathcal{W}^{1, p}(\Omega)^{n}\right)}=\left(\sum_{i=1}^{n} \sum_{j=1}^{N}\left\|\bar{D}_{j, \omega} u_{1, i}\right\|_{L^{p}(Q \times \Omega)}^{p}\right)^{\frac{1}{p}} \text { for } u_{1}=\left(u_{1, i}\right)_{1 \leq i \leq n},
$$

and the corresponding definition for $\left\|u_{2}\right\|_{L^{p}\left(Q \times \Omega ; W_{\#}^{1, p}(Y)^{n}\right)}^{p}$. With this norm, $\mathbb{F}_{0}^{1, p}$ is a Banach space admitting $F_{0}^{\infty}=\left[\mathcal{C}_{0}^{\infty}(Q) \otimes I_{n v}^{p}(\Omega)\right]^{n} \times\left[\mathcal{C}_{0}^{\infty}(Q) \otimes\left[I_{p}\left(\mathcal{C}^{\infty}(\Omega)\right)\right]^{n}\right] \times$ $\left[\mathcal{C}_{0}^{\infty}(Q) \otimes \mathcal{C}^{\infty}(\Omega) \otimes \mathcal{D}_{\#}(Y)\right]^{n}$ as a dense subspace, where $\mathcal{D}_{\#}(Y)=\left\{u \in \mathcal{C}_{\text {per }}^{\infty}(Y)\right.$ : $\left.\int_{Y} u d y=0\right\}$ and $I_{p}$ denote the canonical mapping of $\mathcal{C}^{\infty}(\Omega)$ into its completion $\mathcal{W}^{1, p}(\Omega)$.

Now, for $\mathbf{v}=\left(v_{0}, v_{1}, v_{2}\right) \in \mathbb{F}_{0}^{1, p}$, set $\mathbb{D} \mathbf{v}=D v_{0}+\bar{D}_{\omega} v_{1}+D_{y} v_{2} \in L^{p}(Q \times \Omega \times Y)^{n N}$ and define the functional $F$ on $\mathbb{F}_{0}^{1, p}$ by

$$
F(\mathbf{v})=\iiint_{Q \times \Omega \times Y} f(\cdot, \cdot, \mathbb{D} \mathbf{v}) d x d \mu d y
$$

where the function $f$ here is defined as in Proposition 5. Then due to the strict convexity of $f$, there exists a unique function $\mathbf{u}=\left(u_{0}, u_{1}, u_{2}\right) \in \mathbb{F}_{0}^{1, p}$ that minimizes 
$F$ on $\mathbb{F}_{0}^{1, p}$, i.e.,

$$
F(\mathbf{u})=\inf _{\mathbf{v} \in \mathbb{F}_{0}^{1, p}} F(\mathbf{v}) .
$$

4.3. Regularization and partial results. Let $\theta_{m} \in \mathcal{D}\left(\mathbb{R}^{n N}\right)$ (integer $m \geq 1$ ) with $0 \leq \theta_{m}, \operatorname{supp} \theta_{m} \subset \frac{1}{m} \bar{B}_{n N}$ ( $B_{n N}$ the open unit ball in $\mathbb{R}^{n N}, \bar{B}_{n N}$ its closure in $\mathbb{R}^{n N}$ ) and $\int \theta_{m}(\lambda) d \lambda=1$. We regularize the integrand $f$ in $F_{\varepsilon}$ in order to get an approximating family of integrands $\left(f_{m}\right)_{m \geq 1}$ satisfying standard growth conditions of order $p$ and differentiability as follows:

$f_{m}(x, \omega, y, \lambda)=\int \theta_{m}(\eta) f(x, \omega, y, \lambda-\eta) d \eta\left((x, \omega, y, \lambda) \in \mathbb{R}^{N} \times \Omega \times \mathbb{R}^{N} \times \mathbb{R}^{n N}\right)$.

The main properties of this new integrand are the following:

$\left(\mathrm{H}_{1}\right)_{m}$ We have

$$
\left|f_{m}(x, \omega, y, \lambda)-f_{m}\left(x^{\prime}, \omega, y, \lambda\right)\right| \leq \varpi\left(\left|x-x^{\prime}\right|\right)\left(a(y)+f_{m}(x, \omega, y, \lambda)\right)
$$

for all $x, x^{\prime} \in \mathbb{R}^{N}, \lambda \in \mathbb{R}^{n N}$ and for $d \mu \times d x$-almost all $(\omega, y) \in \Omega \times \mathbb{R}^{N}$, where the functions $\varpi$ and $a$ are as in $\left(\mathrm{H}_{1}\right)$,

$\left(\mathrm{H}_{2}\right)_{m}$ For all $(x, y, \lambda) \in \mathbb{R}^{N} \times \mathbb{R}^{N} \times \mathbb{R}^{n N}$ and for almost all $\omega \in \Omega, f_{m}(x, \cdot, y, \lambda)$ is measurable and $f_{m}(\cdot, \omega, \cdot, \cdot)$ is continuous,

$\left(\mathrm{H}_{3}\right)_{m} f_{m}(x, \omega, y, \cdot)$ is convex for $d \mu \times d x$-almost all $(\omega, y) \in \Omega \times \mathbb{R}^{N}$ and for all $x \in \mathbb{R}^{N}$,

$\left(\mathrm{H}_{4}\right)_{m}$ There exist $0<c_{3} \leq c_{4}, c_{3}$ depending or not on $m$, and $c_{4}$ not depending on $m$, such that

$c_{3}|\lambda|^{p} \leq f_{m}(x, \omega, y, \lambda) \leq c_{4}\left(1+|\lambda|^{p}\right)\left(x \in \mathbb{R}^{N}, \lambda \in \mathbb{R}^{n N}\right.$, a.e. $\left.(\omega, y) \in \Omega \times \mathbb{R}^{N}, m \geq 1\right)$

$\left(\mathrm{H}_{5}\right)_{m} \frac{\partial f_{m}}{\partial \lambda}(x, \omega, y, \lambda)$ exists for all $(x, \lambda)$ and for $d \mu \times d x$-almost all $(\omega, y) \in \Omega \times \mathbb{R}^{N}$, and there exists a constant $c>0$ depending on $m$ such that

$$
\left|\frac{\partial f_{m}}{\partial \lambda}(x, \omega, y, \lambda)\right| \leq c\left(1+|\lambda|^{p-1}\right)
$$

for all $(x, \lambda) \in \mathbb{R}^{N} \times \mathbb{R}^{n N}$ and for almost all $(\omega, y) \in \Omega \times \mathbb{R}^{N}(m \geq 1)$.

Properties $\left(\mathrm{H}_{1}\right)_{m}-\left(\mathrm{H}_{5}\right)_{m}$ easily derive from properties $\left(\mathrm{H}_{1}\right)-\left(\mathrm{H}_{4}\right)$ and from the Riemann-Lebesgue theorem.

Now, let $\Psi \in L^{p}\left(\Omega ; L_{\mathrm{per}}^{p}(Y)\right)^{n N}$; then it is easy to see that the function $\omega \mapsto$ $f_{m}(x, \omega, \cdot, \Psi(\omega, \cdot))$ is measurable from $\Omega$ to $L_{\text {per }}^{1}(Y)$, so that the function $f_{m}(x, \cdot, \cdot, \Psi)$ is an element of $L^{1}\left(\Omega ; L_{\mathrm{per}}^{1}(Y)\right)$. Moreover we have the following

Lemma 2. Let $u \in L^{p}\left(Q \times \Omega ; L_{\mathrm{per}}^{p}(Y)\right)^{n N}$. Then, as $m \rightarrow \infty$,

$$
f_{m}(\cdot, \cdot, u) \rightarrow f(\cdot, \cdot, u) \text { in } L^{1}(Q \times \Omega \times Y) .
$$

Proof. Let $u$ be as above; then

$$
\begin{aligned}
& \left\|f_{m}(\cdot, \cdot, u)-f(\cdot, \cdot, u)\right\|_{L^{1}(Q \times \Omega \times Y)} \\
& =\iiint_{Q \times \Omega \times Y}\left|f_{m}(x, \omega, y, u(x, \omega, y))-f(x, \omega, y, u(x, \omega, y))\right| d x d \mu d y \\
& \leq \iiint_{Q \times \Omega \times Y}\left[\int \theta_{m}(\eta)|f(x, \omega, y, u(x, \omega, y)-\eta)-f(x, \omega, y, u(x, \omega, y))| d \eta\right] d x d \mu d y \\
& \leq c^{\prime} \iiint_{Q \times \Omega \times Y}\left[\int \theta_{m}(\eta)\left[1+|u(x, \omega, y)-\eta|^{p-1}+|u(x, \omega, y)|^{p-1}\right]|\eta| d \eta\right] d x d \mu d y \\
& \leq \frac{c^{\prime \prime}}{m} \iiint_{Q \times \Omega \times Y} \sup _{|\eta| \leq \frac{1}{m}}\left[1+|u(x, \omega, y)-\eta|^{p-1}+|u(x, \omega, y)|^{p-1}\right] d x d \mu d y
\end{aligned}
$$


$c^{\prime}$ and $c^{\prime \prime}$ being independent of $m$. Setting

$H_{m}(x, \omega, y)=\frac{1}{m} \sup _{|\eta| \leq \frac{1}{m}}\left[1+|u(x, \omega, y)-\eta|^{p-1}+|u(x, \omega, y)|^{p-1}\right]$ for $(x, \omega, y) \in Q \times \Omega \times Y$

with $m \geq 1$, we have $H_{m}(x, \omega, y) \rightarrow 0$ as $m \rightarrow \infty$. Thus the result follows by the Lebesgue dominated convergence theorem.

We are now ready to prove one of the most important results of this section.

Proposition 6. Let $\left(v_{\varepsilon}\right)_{\varepsilon \in E}$ be a sequence in $L^{p}(Q \times \Omega)^{n N}$ which stochastically weakly two-scale converges componentwise to $v \in L^{p}\left(Q \times \Omega ; L_{\mathrm{per}}^{p}(Y)\right)^{n N}$. Then for any $m \geq 1$ we have

$\iiint_{Q \times \Omega \times Y} f_{m}(\cdot, \cdot, v) d x d \mu d y \leq \liminf _{E \ni \varepsilon \rightarrow 0} \iint_{Q \times \Omega} f_{m}\left(x, T\left(\frac{x}{\varepsilon_{1}}\right) \omega, \frac{x}{\varepsilon_{2}}, v_{\varepsilon}(x, \omega)\right) d x d \mu$.

Proof. Let $\Psi \in\left(\mathcal{C}_{0}^{\infty}(Q) \otimes \mathcal{C}^{\infty}(\Omega) \otimes \mathcal{C}_{\text {per }}(Y)\right)^{n N}$; since $f_{m}(x, \omega, y, \cdot)$ is convex and differentiable we have

$$
\begin{gathered}
\iint_{Q \times \Omega} f_{m}\left(x, T\left(\frac{x}{\varepsilon_{1}}\right) \omega, \frac{x}{\varepsilon_{2}}, v_{\varepsilon}(x, \omega)\right) d x d \mu \\
\geq \iint_{Q \times \Omega} f_{m}\left(x, T\left(\frac{x}{\varepsilon_{1}}\right) \omega, \frac{x}{\varepsilon_{2}}, \Psi\left(x, T\left(\frac{x}{\varepsilon_{1}}\right) \omega, \frac{x}{\varepsilon_{2}}\right)\right) d x d \mu \\
+\iint_{Q \times \Omega} \frac{\partial f_{m}}{\partial \lambda}\left(x, T\left(\frac{x}{\varepsilon_{1}}\right) \omega, \frac{x}{\varepsilon_{2}}, \Psi\left(x, T\left(\frac{x}{\varepsilon_{1}}\right) \omega, \frac{x}{\varepsilon_{2}}\right)\right) \cdot\left(v_{\varepsilon}(x, \omega)-\Psi\left(x, T\left(\frac{x}{\varepsilon_{1}}\right) \omega, \frac{x}{\varepsilon_{2}}\right)\right) d x d \mu .
\end{gathered}
$$

Since the function $f_{m}(x, \cdot, \cdot, \Psi(x, \cdot, \cdot)$,$) is an element of L^{\infty}\left(\Omega ; L_{\text {per }}^{\infty}(Y)\right)$ we have the convergence result

$$
\begin{aligned}
& \lim _{E \ni \varepsilon \rightarrow 0} \iint_{Q \times \Omega} f_{m}\left(x, T\left(\frac{x}{\varepsilon_{1}}\right) \omega, \frac{x}{\varepsilon_{2}}, \Psi\left(x, T\left(\frac{x}{\varepsilon_{1}}\right) \omega, \frac{x}{\varepsilon_{2}}\right)\right) d x d \mu \\
= & \iiint_{Q \times \Omega \times Y} f_{m}(x, \omega, y, \Psi(x, \omega, y)) d x d \mu d y .
\end{aligned}
$$

On the other hand, the function $\frac{\partial f_{m}}{\partial \lambda_{i}}(\cdot, \cdot, \Psi) \in \mathcal{C}\left(\bar{Q} ; L^{\infty}\left(\Omega ; L_{\text {per }}^{\infty}(Y)\right)\right)$ where $\frac{\partial f_{m}}{\partial \lambda_{i}}(\cdot, \cdot, \Psi)$ stands for the function $x \mapsto \frac{\partial f_{m}}{\partial \lambda_{i}}(x, \cdot, \cdot, \Psi(x, \cdot, \cdot))$ from $\bar{Q}$ to $L^{\infty}\left(\Omega ; L_{\mathrm{per}}^{\infty}(Y)\right)$, so that, as in the framework of deterministic homogenization (see particularly [25]) one can take this function as a test function in (3.1) (see Definition 1) and get, as $E \ni \varepsilon \rightarrow 0$,

$$
\begin{aligned}
& \iint_{Q \times \Omega} \frac{\partial f_{m}}{\partial \lambda}\left(x, T\left(\frac{x}{\varepsilon_{1}}\right) \omega, \frac{x}{\varepsilon_{2}}, \Psi\left(x, T\left(\frac{x}{\varepsilon_{1}}\right) \omega, \frac{x}{\varepsilon_{2}}\right)\right) \cdot\left(v_{\varepsilon}(x, \omega)-\Psi\left(x, T\left(\frac{x}{\varepsilon_{1}}\right) \omega, \frac{x}{\varepsilon_{2}}\right)\right) d x d \mu \\
& \rightarrow \iiint_{Q \times \Omega \times Y} \frac{\partial f_{m}}{\partial \lambda}(x, \omega, y, \Psi(x, \omega, y)) \cdot(v(x, \omega, y)-\Psi(x, \omega, y)) d x d \mu d y .
\end{aligned}
$$

Therefore

$\liminf _{E \ni \varepsilon \rightarrow 0} \iint_{Q \times \Omega} f_{m}\left(x, \frac{x}{\varepsilon}, v_{\varepsilon}(x)\right) d x d \mu \geq \iiint_{Q \times \Omega \times Y} f_{m}(x, \omega, y, \Psi(x, \omega, y)) d x d \mu d y$

$+\iiint_{Q \times \Omega \times Y} \frac{\partial f_{m}}{\partial \lambda}(x, \omega, y, \Psi(x, \omega, y)) \cdot(v(x, \omega, y)-\Psi(x, \omega, y)) d x d \mu d y$.

But, we have to take into account property $\left(\mathrm{H}_{m}\right)_{5}$ to get

$$
\begin{aligned}
& \left|\iiint_{Q \times \Omega \times Y} \frac{\partial f_{m}}{\partial \lambda}(x, \omega, y, \Psi(x, \omega, y)) \cdot(v(x, \omega, y)-\Psi(x, \omega, y)) d x d \mu d y\right| \\
& \quad \leq 2^{\frac{1}{p}} c^{\frac{1}{p^{\prime}}}\left[|Q|+\|\Psi\|_{L^{p}(Q \times \Omega \times Y)^{n N}}^{p}\right]^{\frac{1}{p^{\prime}}}\|v-\Psi\|_{L^{p}(Q \times \Omega \times Y)^{n N}} .
\end{aligned}
$$

Thus, by choosing a sequence $\left(\Psi_{l}\right)_{l}$ in $\left(\mathcal{C}_{0}^{\infty}(Q) \otimes \mathcal{C}^{\infty}(\Omega) \otimes \mathcal{C}_{\text {per }}(Y)\right)^{n N}$ such that $\Psi_{l} \rightarrow v$ in $L^{p}(Q \times \Omega \times Y)^{n N}$ as $l \rightarrow \infty$, it follows that for fixed $\delta>0$, there exists 
$l_{0} \in \mathbb{N}$ such that

$$
\left|\iiint_{Q \times \Omega \times Y} \frac{\partial f_{m}}{\partial \lambda}(x, \omega, y, \Psi(x, \omega, y)) \cdot(v(x, \omega, y)-\Psi(x, \omega, y)) d x d \mu d y\right| \leq \delta
$$

for all $l \geq l_{0}$. Hence

$$
\liminf _{E \ni \varepsilon \rightarrow 0} \iint_{Q \times \Omega} f_{m}^{\varepsilon}\left(\cdot, \cdot, v_{\varepsilon}\right) d x d \mu \geq \iiint_{Q \times \Omega \times Y} f_{m}\left(\cdot, \cdot, \Psi_{l}\right) d x d \mu d y-\delta
$$

for all $l \geq l_{0}$. Passing to the limit as $l \rightarrow \infty$, and using the continuity of $\lambda \mapsto$ $f_{m}(\cdot, \cdot, \lambda)$ yields

$$
\liminf _{E \ni \varepsilon \rightarrow 0} \iint_{Q \times \Omega} f_{m}^{\varepsilon}\left(\cdot, \cdot, v_{\varepsilon}\right) d x d \mu \geq \iiint_{Q \times \Omega \times Y} f_{m}(\cdot, \cdot, v) d x d \mu d y-\delta .
$$

Since $\delta$ is arbitrarily fixed, we are led at once to the result by letting $\delta \rightarrow 0$.

It results from the preceding proposition the following

Corollary 2. Let $\left(u_{\varepsilon}\right)_{\varepsilon \in E}$ be a sequence in $L^{p}\left(\Omega ; W^{1, p}\left(Q ; \mathbb{R}^{n}\right)\right)$. Assume that $\left(D u_{\varepsilon}\right)_{\varepsilon \in E}$ stochastically weakly two-scale converges componentwise to $\mathbb{D} \mathbf{u}=D u_{0}+$ $\bar{D}_{\omega} u_{1}+D_{y} u_{2} \in L^{p}\left(Q \times \Omega ; L_{\mathrm{per}}^{p}(Y)\right)^{n N}$, where $\mathbf{u}=\left(u_{0}, u_{1}, u_{2}\right) \in \mathbb{F}_{0}^{1, p}$. Then

$$
\iiint_{Q \times \Omega \times Y} f(\cdot, \cdot, \mathbb{D} \mathbf{u}) d x d \mu d y \leq \liminf _{E \ni \varepsilon \rightarrow 0} \iint_{Q \times \Omega} f^{\varepsilon}\left(\cdot, \cdot, D u_{\varepsilon}\right) d x d \mu
$$

Proof. Let $\eta \in \mathbb{R}^{n N}$; then

$$
f(x, \omega, y, \lambda-\eta) \leq f(x, \omega, y, \lambda)+c_{2}\left(1+|\lambda-\eta|^{p-1}+|\lambda|^{p-1}\right)|\eta| \text { (see (4.4)), }
$$

hence

$$
f_{m}(x, \omega, y, \lambda) \leq f(x, \omega, y, \lambda)+c_{2} \int \theta_{m}(\eta)\left(1+|\lambda-\eta|^{p-1}+|\lambda|^{p-1}\right)|\eta| d \eta .
$$

Thus

$$
f_{m}(x, \omega, y, \lambda) \leq f(x, \omega, y, \lambda)+\frac{c_{2}}{m} \sup _{|\eta| \leq \frac{1}{m}}\left(1+|\lambda-\eta|^{p-1}+|\lambda|^{p-1}\right),
$$

so that

$$
\begin{aligned}
& \iint_{Q \times \Omega} f_{m}\left(x, T\left(\frac{x}{\varepsilon_{1}}\right) \omega, \frac{x}{\varepsilon_{2}}, D u_{\varepsilon}(x, \omega)\right) d x d \mu \\
& \quad \leq \iint_{Q \times \Omega} f\left(x, T\left(\frac{x}{\varepsilon_{1}}\right) \omega, \frac{x}{\varepsilon_{2}}, D u_{\varepsilon}(x, \omega)\right) d x d \mu \\
& \quad+\frac{c_{2}}{m} \iint_{Q \times \Omega}\left(\sup _{|\eta| \leq \frac{1}{m}}\left(1+\left|D u_{\varepsilon}(x, \omega)-\eta\right|^{p-1}+\left|D u_{\varepsilon}(x, \omega)\right|^{p-1}\right)\right) d x d \mu .
\end{aligned}
$$

Therefore, using Proposition 6 we get

$$
\begin{aligned}
& \iiint_{Q \times \Omega \times Y} f_{m}(\cdot, \cdot, \mathbb{D} \mathbf{u}) d x d \mu d y \leq \liminf _{E \ni \varepsilon \rightarrow 0} \iint_{Q \times \Omega} f_{m}\left(x, T\left(\frac{x}{\varepsilon_{1}}\right) \omega, \frac{x}{\varepsilon_{2}}, D u_{\varepsilon}(x, \omega)\right) d x d \mu \\
& \leq \liminf _{E \ni \varepsilon \rightarrow 0} \iint_{Q \times \Omega} f\left(x, T\left(\frac{x}{\varepsilon_{1}}\right) \omega, \frac{x}{\varepsilon_{2}}, D u_{\varepsilon}(x, \omega)\right) d x d \mu+ \\
& +\frac{c_{2}}{m} \liminf _{E \ni \varepsilon \rightarrow 0} \iint_{Q \times \Omega}\left(\sup _{|\eta| \leq \frac{1}{m}}\left(1+\left|D u_{\varepsilon}(x, \omega)-\eta\right|^{p-1}+\left|D u_{\varepsilon}(x, \omega)\right|^{p-1}\right)\right) d x d \mu .
\end{aligned}
$$

By letting $m \rightarrow \infty$, using Lemma 2 we are led to (4.12). 
4.4. Homogenization result. We are now able to prove the main homogenization result of this section.

Theorem 3. Let $1<p<\infty$. For each real $\varepsilon>0$, let $u_{\varepsilon}$ be the unique solution of (4.6). Then, as $\varepsilon \rightarrow 0$,

$$
u_{\varepsilon} \rightarrow u_{0} \text { stoch. in } L^{p}(Q \times \Omega)^{n} \text {-weak (see (3.3)) }
$$

and

$$
D u_{\varepsilon} \rightarrow D u_{0}+\bar{D}_{\omega} u_{1}+D_{y} u_{2} \text { stoch. in } L^{p}(Q \times \Omega)^{n N} \text {-weak } 2 s
$$

where $\mathbf{u}=\left(u_{0}, u_{1}, u_{2}\right) \in \mathbb{F}_{0}^{1, p}$ is the unique solution to the minimization problem (4.11).

Proof. In view of the growth conditions in $\left(\mathrm{H}_{4}\right)$, the sequence $\left(u_{\varepsilon}\right)_{\varepsilon>0}$ is bounded in $L^{p}\left(\Omega ; W_{0}^{1, p}\left(Q ; \mathbb{R}^{n}\right)\right)$ and so the sequence $\left(f^{\varepsilon}\left(\cdot, \cdot, D u_{\varepsilon}\right)\right)_{\varepsilon>0}$ is bounded in $L^{1}(Q \times$ $\Omega$ ). Thus, given an arbitrary fundamental sequence $E$, we get by Theorem 2 the existence of a subsequence $E^{\prime}$ from $E$ and a triple $\mathbf{u}=\left(u_{0}, u_{1}, u_{2}\right) \in \mathbb{F}_{0}^{1, p}$ such that (4.13)-(4.14) hold when $E^{\prime} \ni \varepsilon \rightarrow 0$. The sequence $\left(F_{\varepsilon}\left(u_{\varepsilon}\right)\right)_{\varepsilon>0}$ consisting of real numbers being bounded (since $\left(u_{\varepsilon}\right)_{\varepsilon>0}$ is bounded in $L^{p}\left(\Omega ; W_{0}^{1, p}\left(Q ; \mathbb{R}^{n}\right)\right)$ ), there exists a subsequence from $E^{\prime}$ not relabeled such that $\lim _{E^{\prime} \ni \varepsilon \rightarrow 0} F_{\varepsilon}\left(u_{\varepsilon}\right)$ exists. It remains to verify that $\mathbf{u}=\left(u_{0}, u_{1}, u_{2}\right)$ solves (4.11). In fact, if $\mathbf{u}$ solves this problem, then thanks to the uniqueness of the solution of (4.11), the whole sequence $\left(u_{\varepsilon}\right)_{\varepsilon>0}$ will verify (4.13) and (4.14) when $\varepsilon \rightarrow 0$. Thus our only concern here is to check that $\mathbf{u}$ solves problem (4.11). To this end, in view of Corollary 2, we have

$$
\iiint_{Q \times \Omega \times Y} f(\cdot, \cdot, \mathbb{D} \mathbf{u}) d x d \mu d y \leq \lim _{E^{\prime} \ni \varepsilon \rightarrow 0} \iint_{Q \times \Omega} f\left(x, T\left(\frac{x}{\varepsilon_{1}}\right) \omega, \frac{x}{\varepsilon_{2}}, D u_{\varepsilon}(x, \omega)\right) d x d \mu .
$$

On the other hand, let us establish an upper bound for

$$
\iint_{Q \times \Omega} f\left(x, T\left(\frac{x}{\varepsilon_{1}}\right) \omega, \frac{x}{\varepsilon_{2}}, D u_{\varepsilon}(x, \omega)\right) d x d \mu .
$$

To do that, let $\Phi=\left(\psi_{0}, I_{p}\left(\psi_{1}\right), \psi_{2}\right) \in F_{0}^{\infty}$ with $\psi_{0} \in\left[\mathcal{C}_{0}^{\infty}(Q) \otimes I_{n v}^{p}(\Omega)\right]^{n}, \psi_{1} \in$ $\left[\mathcal{C}_{0}^{\infty}(Q) \otimes \mathcal{C}^{\infty}(\Omega)\right]^{n}$ and $\psi_{2} \in\left[\mathcal{C}_{0}^{\infty}(Q) \otimes \mathcal{C}^{\infty}(\Omega) \otimes \mathcal{D}_{\#}(Y)\right]^{n}$. Define $\Phi_{\varepsilon}$ as in Corollary 1 (see (4.9) therein). Since $u_{\varepsilon}$ is the minimizer, one has

$$
\iint_{Q \times \Omega} f\left(x, T\left(\frac{x}{\varepsilon_{1}}\right) \omega, \frac{x}{\varepsilon_{2}}, D u_{\varepsilon}(x, \omega)\right) d x d \mu \leq \iint_{Q \times \Omega} f\left(x, T\left(\frac{x}{\varepsilon_{1}}\right) \omega, \frac{x}{\varepsilon_{2}}, D \Phi_{\varepsilon}(x, \omega)\right) d x d \mu
$$

Thus, using Corollary 1 we get

$$
\begin{aligned}
\lim _{E^{\prime} \ni \varepsilon \rightarrow 0} & \iint_{Q \times \Omega} f\left(x, T\left(\frac{x}{\varepsilon_{1}}\right) \omega, \frac{x}{\varepsilon_{2}}, D u_{\varepsilon}(x, \omega)\right) d x d \mu \\
& \leq \iiint_{Q \times \Omega \times Y} f\left(\cdot, \cdot, D \psi_{0}+D_{\omega} \psi_{1}+D_{y} \psi_{2}\right) d x d \mu d y
\end{aligned}
$$

for any $\Phi \in F_{0}^{\infty}$, and by density, for all $\Phi$ in $\mathbb{F}_{0}^{1, p}$. From which we get

$$
\lim _{E^{\prime} \ni \varepsilon \rightarrow 0} \iint_{Q \times \Omega} f\left(x, T\left(\frac{x}{\varepsilon_{1}}\right) \omega, \frac{x}{\varepsilon_{2}}, D u_{\varepsilon}(x, \omega)\right) d x d \mu \leq \inf _{\mathbf{v} \in \mathbb{F}_{0}^{1, p}} \iiint_{Q \times \Omega \times Y} f(\cdot, \cdot, \mathbb{D} \mathbf{v}) d x d \mu d y .
$$

Inequalities (4.15) and (4.16) yield

$$
\iiint_{Q \times \Omega \times Y} f(\cdot, \cdot, \mathbb{D} \mathbf{u}) d x d \mu d y=\inf _{\mathbf{v} \in \mathbb{F}_{0}^{1, p}} \iiint_{Q \times \Omega \times Y} f(\cdot, \cdot, \mathbb{D} \mathbf{v}) d x d \mu d y
$$


i.e., (4.11). The proof is complete.

In order to derive the macroscopic homogenized problem, we need to assume that the dynamical system $T(x)$ is ergodic. Assuming this, the function $u_{0}$ (in Theorem 3) does not depend on the random variable $\omega$ since in this case we have $I_{n v}^{p}(\Omega)$ consists of constants. With this in mind, let

$$
f_{\text {hom }}^{1}(x, \omega, \lambda)=\int_{Y} f\left(x, \omega, y, \lambda+D_{y} \pi_{1}\left(x, \omega, D u_{0}(x)+\bar{D}_{\omega} u_{1}(x, \omega)\right)\right) d y
$$

where, thanks to the strict convexity and to the bounds (4.3) satisfied by the function $f$, the function $\pi_{1}(x, \omega, \xi)$ (for fixed $\left.(x, \omega, \xi)\right)$ is the unique solution to the variational problem

$$
\int_{Y} f\left(x, \omega, y, \xi+D_{y} \pi_{1}(x, \omega, \xi)\right) d y=\inf _{\phi \in W_{\#}^{1, p}(Y)^{n}} \int_{Y} f\left(x, \omega, y, \xi+D_{y} \phi\right) d y,
$$

and where the function $(x, \omega) \mapsto \pi_{1}\left(x, \omega, D u_{0}(x)+\bar{D}_{\omega} u_{1}(x, \omega)\right)$ from $Q \times \Omega$ to $W_{\#}^{1, p}(Y)^{n}$, lies in $L^{p}\left(Q \times \Omega ; W_{\#}^{1, p}(Y)^{n}\right)$. Next, set

$$
f_{\text {hom }}(x, \lambda)=\int_{\Omega} f_{\text {hom }}^{1}\left(x, \omega, \lambda+\bar{D}_{\omega} \pi_{2}\left(x, D u_{0}(x)\right)\right) d \mu
$$

where, as above, the function $\pi_{2}(x, \xi)$ (for fixed $(x, \xi)$ ) is the unique solution to the variational cell problem

$$
\int_{\Omega} f_{\text {hom }}^{1}\left(x, \omega, \xi+\bar{D}_{\omega} \pi_{2}(x, \xi)\right) d \mu=\inf _{\phi \in \mathcal{W}^{1, p}(\Omega)^{n}} \int_{\Omega} f_{\text {hom }}^{1}\left(x, \omega, \xi+\bar{D}_{\omega} \phi\right) d \mu .
$$

and where also, the function $x \mapsto \pi_{2}\left(x, D u_{0}(x)\right)$ from $Q$ to $\mathcal{W}^{1, p}(\Omega)^{n}$, lies in $L^{p}\left(Q ; \mathcal{W}^{1, p}(\Omega)^{n}\right)$. Then $u_{0}$ is the unique solution to the variational problem

$$
\int_{Q} f_{\text {hom }}\left(x, D u_{0}(x)\right) d x=\inf _{v \in W_{0}^{1, p}(Q)^{n}} \int_{Q} f_{\text {hom }}(x, D v(x)) d x .
$$

Therefore one can argue that our results and the classical results in the literature in some well-known situations (see, e.g., $[5,6,11]$ ) are equivalent when the dynamical system is ergodic.

Remark 4. We see that in the case where the dynamical system is ergodic, the macroscopic homogenized equation is a deterministic one.

Acknowledgement 1. The authors thank the anonymous referee for his/her helpful comments and suggestions. The work of the second author is supported by the University of Pretoria through a postdoctoral fellowship. He also acknowledges the support of the National Research Foundation of South Africa.

\section{REFERENCES}

[1] Y. Abddaimi, G. Michaille and C. Licht, Stochastic homogenization for an integral functional of quasiconvex function with linear growth, Asymptotic Anal. 15 (1997), 183-202.

[2] G. Allaire, Homogenization and two-scale convergence, SIAM J. Math. Anal. 23 (1992), 1482-1518.

[3] G. Allaire and M. Briane, Multi-scale convergence and reiterated homogenization, Proc. Roy. Soc. Edinb. Sect. A. 126 (1996), 297-342.

[4] K.T. Andrews and S. Wright, Stochastic homogenization of elliptic boundary-value problems with $L^{p}$-data, Asymptotic Anal. 17 (1998), 165-184.

[5] M. Baia and I. Fonseca, $\Gamma$-convergence of functionals with periodic integrands via 2-scale convergence, Technical Report, 2005. 
[6] M. Barchiesi, Multiscale homogenization of convex functionals with discontinuous integrand, J. Convex Anal. 14 (2007), 205-226.

[7] X. Blanc, C. Le Bris and P.L. Lions, Stochastic homogenization and random lattices, J. Math. Pures Appl. 88 (2007), 34-63.

[8] N. Bourbaki, Topologie générale, Chap. 1-4, Hermann, Paris, 1971.

[9] N. Bourbaki, Intégration, Chap. 1-4, Hermann, Paris, 1966.

[10] A. Bourgeat, A. Mikelic and S. Wright, Stochastic two-scale convergence in the mean and applications, J. Reine Angew. Math. 456 (1994), 19-51.

[11] A. Braides and D. Lukkassen, Reiterated homogenization of integral functionals, Math. Mod. Meth. Appl. Sci. 10 (2000), 47-71.

[12] C. Castaings and M. Valadier, Convex analysis and measurable multifunctions, Lecture Notes in Math., 580, Springer, 1977.

[13] G. Dal Maso and L. Modica, Nonlinear stochastic homogenization, Ann. Mat. Pura Appl. 144 (1986), 347-389.

[14] G. Dal Maso and L. Modica, Nonlinear stochastic homogenization and ergodic theory, J. Reine Angew. Math. 368 (1986), 28-42.

[15] N. Dunford and J.T. Schwartz, Linear operators, Parts I and II, Interscience Publishers, Inc., New York, 1958, 1963.

[16] I. Ekeland and R. Temam, Convex analysis and variational problems, Vol.I, Studies in Math. and its Applications, North-Holland, Amsterdam, 1976.

[17] B. Gambin and J.J. Telega, Effective properties of elastic solids with with randomly distributed microcracks, Mech. Res. Comm. 27 (2000), 697-706.

[18] V.V. Jikov, S.M. Kozlov and O.A. Oleinik, Homogenization of differential operators and integral functionals, Springer-Verlag, Berlin, 1994.

[19] R. Larsen, Banach algebras, Marcel Dekker, New York, 1973.

[20] D. Lukkassen, G. Nguetseng and P. Wall, Two-scale convergence, Int. J. Pure Appl. Math. 1 (2002), 35-86.

[21] P. Marcellini, Periodic solutions and homogenization of nonlinear variational problems, Ann. Mat. Pura Appl. 117 (1978), 139-152.

[22] K. Messaoudi and G. Michaille, Stochastic homogenization of nonconvex integral functionals, Math. Modelling. Numer. Anal. 28 (1994), 329-356.

[23] G. Nguetseng, A general convergence result for a functional related to the theory of homogenization, SIAM J. Math. Anal. 20 (1989), 608-623.

[24] G. Nguetseng, Homogenization structures and applications I, Z. Anal. Anwen. 22 (2003), 73-107.

[25] G. Nguetseng, H. Nnang and J.L. Woukeng, Deterministic homogenization of integral functionals with convex integrands, Nonlinear Differ. Equ. Appl. NoDEA 17 (2010), 757-781.

[26] G. Nguetseng, M. Sango and J.L. Woukeng, Reiterated ergodic algebras and applications, Commun. Math. Phys. 300 (2010), 835-876.

[27] G. Nguetseng and J.L. Woukeng, Deterministic homogenization of parabolic monotone operators with time dependent coefficients, Electron. J. Differ. Equ. 2004 (2004), 1-23.

[28] A. Pankov, G-convergence and homogenization of nonlinear partial differential operators, Kluwer Academic Publishers, Dordrecht, 1997.

[29] M. Reed, B. Simon, Methods of modern mathematical physics, Vol. I, Functional analysis, Academic Press, New York, 1980.

[30] W. Rudin, Functional analysis, McGraw-Hill, New York, 1973.

[31] K. Sab, Homogenization of non-linear random media by a duality method. Application to plasticity, Asymptotic Anal. 9 (1994), 311-336.

[32] J.J. Telega, Stochastic homogenization: Convexity and nonconvexity, in: P. Ponte Castañeda et al., ed., Nonlinear homogenization and its applications to composite, polycrystals and smart materials, Kluwer Acad. Publishers, Netherlands, (2004), 305-347.

[33] J.J. Telega and W. Bielski, Stochastic homogenization and macrsocopic modelling of composites and flow through porous media, Theoret. Appl. Mech. 28-29 (2002), 337-377.

[34] J.J. Telega and R. M. Stanċzyk, Modelling of soft tissues behaviour, in: Modelling in Biomechanics, ed., J.J. Telega AMAS Lecture notes 19, 2005.

[35] S. Torquado, Random heterogeneous materials: microstructure and macroscopic properties, Springer, New York, 2002.

[36] J. Van Tiel, Convex analysis: An introduction text, Wiley, New York, 1984. 
[37] J.L. Woukeng, Periodic homogenization of nonlinear non-monotone parabolic operators with three time scales, Ann. Mat. Pura Appl. 189 (2010), 357-379.

[38] V.V. Zikhov, On a homogenization technique for variational problems, Funct. Anal. Appl. 33 (1999), 11-24.

[39] V.V. Zhikov, On two-scale convergence, J. Math. Sci. 120 (2004), 1328-1352.

[40] V.V. Zhikov S.M. Kozlov and O.A. Oleinik, Averaging of parabolic operators, Trans. Moscow Math. Soc. 45 (1984), 189-241.

[41] V.V. Zhikov and A.L. Pyatnitskii, Homogenization of random singular structures and random measures, Izv.: Mathematics $\mathbf{7 0}$ (2006), 19-67.

M. Sango, Department of Mathematics and Applied Mathematics, University of Pretoria, Pretoria 0002, South Africa

E-mail address: mamadou.sango@up.ac.za

J.L. Woukeng, Department of Mathematics and Computer Science, University of Dschang, P.O. Box 67, Dschang, Cameroon

Current address: J.L. Woukeng (Corresponding author), Department of Mathematics and Applied Mathematics, University of Pretoria, Pretoria 0002, South Africa

E-mail address: jwoukeng@yahoo.fr, jeanlouis.woukeng@up.ac.za 